\title{
Title: Loss of Piccolo function in rats induces Pontocerebellar Hypoplasia type 3-like phenotypes
}

Abbreviated title: Piccolo loss of function triggers $\mathrm{PCH} 3$ phenotypes

\author{
Author names and affiliations: \\ Joanne Falck ${ }^{1}$, Christine Bruns ${ }^{1}$, Sheila Hoffmann ${ }^{1}$, Isabelle Straub ${ }^{2}$, Erik J. Plautz ${ }^{3}$, Marta \\ Orlando $^{4}$, Humaira Munawar ${ }^{5}$, Marion Rivalan ${ }^{4,5}$, York Winter ${ }^{4,5}$, Zsuzsanna Izsvák ${ }^{6}$, Dietmar \\ Schmitz $^{4}$, F. Kent Hamra ${ }^{7}$, Stefan Hallermann ${ }^{2}$, Craig Garner ${ }^{1 *}$ and Frauke Ackermann ${ }^{1 *}$
}

1) German Center for Neurodegenerative Diseases (DZNE), Charité Medical University, Charitéplatz 1, 10117 Berlin, Germany

2) Carl-Ludwig Institute for Physiology, 04103, Leipzig, Germany

3) Department of Neurology and Neurotherapeutics, University of Texas Southwestern, Dallas Texas 75390

4) Charité Medical University Berlin, corporate member of Free University Berlin and Humboldt University Berlin; Berlin Institute of Health, NeuroCure Cluster of Excellence, Charitéplatz 1, 10117 Berlin, Germany

5) Department of Biology, Humboldt University, Philippstr. 13, 10099 Berlin, Germany

6) Max Delbrück Center for Molecular Medicine in the Helmholtz Society, 13125, Berlin, Germany

7) Department of Obstetrics and Gynecology, Cecil H. \& Ida Green Center for Reproductive Biology Sciences, University of Texas Southwestern, Dallas Texas 75390

*equal contribution 
Corresponding author email address: Frauke Ackermann: frauke.ackermann@dzne.de and Craig Garner: craig.garner@dzne.de, German Center for Neurodegenerative Diseases (DZNE), Charité Medical University, Charitéplatz 1, 10117 Berlin, Germany.

Conflict of interest statement: The authors declare no competing financial interests.

\section{Number of pages: 46}

\section{Number of figures: 9}

\section{Number of words: abstract 248, introduction 609, discussion 1499}

Acknowledgments: We would like to thank Susanne Wegmann and Eckart Gundelfinger for discussion and valuable comments on the manuscript; Anny Kretschmer and Katja Czieselsky for technical assistance. The work was supported by German Center for Neurodegenerative Diseases (DZNE), the Federal Government of Germany (DFG) SFB958 to CCG and (DFG) EXC 257 for the Center of Excellence NeuroCure to YW. Work to generate Pclo ${ }^{g t / g t}$ rats was supported by National institute of Health R24RR03232601 \& R24OD011108 to FKH. Neurological analyses on Piccolo mutant rats were conducted by The Neuro-Models Facility (EJP, LBG) at UT Southwestern Medical Center, and supported by the Haggerty Center for Brain Injury and Repair.

Author contributions: J. Falck performed the majority of the experiments and analyzed data. J. Falck, F. Ackermann, F. K. Hamra, S. Hallermann and C. C. Garner designed experiments. M. Orlando performed electron microscopy studies, I. Straub performed electrophysiology experiments and C. Bruns performed Western Blot studies and a portion of the immunohistochemical stainings. Behavioral experiments were performed by E. J. Plautz; Behavioral experiments were performed by E. J. Plautz. and F. K. Hamra. Behavioral experiments and analysis in the OptiMan setup were performed by H. Munawar, M. Rivalan and 
bioRxiv preprint doi: https://doi.org/10.1101/774422; this version posted September 25, 2019. The copyright holder for this preprint (which was not certified by peer review) is the author/funder. All rights reserved. No reuse allowed without permission.

Y. Winter, Z. Izsvák generated $P c l o^{g t / g t}$ rat. J. Falck, S. Hoffmann, C.C. Garner and F. Ackermann wrote the manuscript. 


\section{Abstract}

Piccolo, a presynaptic active zone protein, is best known for its role in the regulated

3 assembly and function of vertebrate synapses. Genetic studies suggest a further link to several

4 psychiatric disorders as well as Pontocerebellar Hypoplasia type 3 (PCH3), although a causal

5 relationship is lacking. We have characterized recently generated knockout $\left(\right.$ Pclo $\left.^{g t / g t}\right)$ rats.

6 Analysis revealed a dramatic reduction in brain size compared to wildtype $\left(\right.$ Pclo $\left.^{w t / w t}\right)$ animals,

7 attributed to a decrease in the size of the cerebral cortical, cerebellar and pontine regions.

8 Analysis of the cerebellum and brainstem revealed a reduced granule cell (GC) layer and a

9 reduction in size of pontine nuclei. Moreover, the maturation of mossy fiber (MF) afferents from

10 pontine neurons and the expression of the $\alpha 6 \mathrm{GABA}_{\mathrm{A}}$ receptor subunit at the MF-GC synapse are

11 perturbed, as well as the innervation of Purkinje cells by cerebellar climbing fibers (CFs).

12 Ultrastructural and functional studies revealed a reduced size of MF boutons, with fewer synaptic

13 vesicles and altered synaptic transmission. These data imply that Piccolo is required for the

14 normal development, maturation and function of neuronal networks formed between the

15 brainstem and cerebellum. Consistently, behavioral studies demonstrated that adult Pclo ${ }^{\text {gt/gt }}$ rats

16 display impaired motor coordination, despite adequate performance in tasks that reflect muscle

17 strength and locomotion. Together these data suggest that loss of Piccolo function in patients with

18 PCH3 could be causal for many of the observed anatomical and behavioral symptoms, and that

19 the further analysis of these animals could provide fundamental mechanistic insights into this

20 devastating disorder.

22 Key Words: presynapse, active zone, Piccolo, Pontocerebellar hypoplasia

24 Significance Statement: Pontocerebellar Hypoplasia type 3 is a devastating developmental

25 disorder associated with severe developmental delay, progressive microcephaly with

26 brachycephaly, optic atrophy, seizures and hypertonia with hyperreflexia. Recent genetic studies 
27 have identified non-sense mutations in the coding region of the Piccolo gene, suggesting a

28 functional link between this disorder and the presynaptic active zone. Our analysis of Piccolo

29 knockout rats supports this hypothesis, formally demonstrating that anatomical and behavioral

30 phenotypes seen in patients with $\mathrm{PCH} 3$ are also exhibited by these Piccolo deficient animals.

\section{Introduction}

33 Pontocerebellar Hypoplasia is a rare and highly heterogeneous group of neurological

34 disorders, often with a genetic origin, characterized by an abnormally small cerebellum and pons

35 (Rajab et al., 2003; Namavar et al., 2011). In type 3 Pontocerebellar Hypoplasia (PCH3) - also

36 known as Cerebellar Atrophy with Progressive Microcephaly (CLAM) - patients suffer from

37 severe developmental delay, progressive microcephaly with brachycephaly, seizures, hypertonia

38 with hyperreflexia and short stature (Rajab et al., 2003; Namavar et al., 2011). Additional features

39 include the presence of craniofacial dysmorphisms and optic atrophy (Durmaz et al., 2009;

40 Rudnik-Schoneborn et al., 2014).

41 Previous studies mapped the PCH phenotype to chromosome 7q11-21 (Rajab et al., 2003;

42 Durmaz et al., 2009). More recently, a single nucleotide polymorphism (SNP) in the human Pclo

43 gene - indeed, located on chromosome 7 at position 21.11 - has been found in patients with

$44 \mathrm{PCH} 3$. This non-sense mutation is predicted to eliminate the C-terminus of the longest Piccolo

45 isoforms including its PDZ and C2 domains (Ahmed et al., 2015) and perhaps destabilize the

46 protein, leading to the hypothesis that Piccolo loss of function is responsible for the phenotypes

47 seen in this neurodevelopmental/neurodegenerative disorder.

48 Piccolo is a very large $(560 \mathrm{kDa})$ multidomain presynaptic scaffold protein and core

49 component of the cytoskeletal matrix assembled at active zones (CAZ) (Cases-Langhoff et al.,

50 1996). A range of studies suggest that Piccolo uses its multidomain structure to scaffold not only

51 other CAZ proteins critical for the regulated release of neurotransmitters, but also proteins

52 involved in the dynamic assembly of F-actin, synaptic vesicle (SV) recycling and synapse 
53 integrity (Gundelfinger et al., 2015; Ackermann et al., 2019). Intriguingly, Piccolo is present at

54 nearly every synaptic subtype including glutamatergic, GABAergic, cholinergic and

55 dopaminergic synapses within the central (CNS) and peripheral nervous system (PNS) (Cases-

56 Langhoff et al., 1996; Fenster et al., 2000; Fenster and Garner, 2002) and is highly expressed in

57 the cerebrum, hippocampus, cerebellum and olfactory bulb, among others (Cases-Langhoff et al.,

58 1996; Human Protein Atlas, 2015). It is one of the very first active zone (AZ) proteins recruited

59 to nascent synapses in vitro as well as in the developing brain (Zhai et al., 2001). For example,

60 Piccolo appears at emerging synapses formed between mossy fiber boutons and cerebellar

61 granule cells as well as between parallel fiber boutons and Purkinje cell dendrites during the

62 earliest stages of cerebellar development (Zhai et al., 2001). The large size of Piccolo and the

63 complexity of the Pclo gene has thwarted most efforts to elucidate its function, though critical

64 roles in retinal ribbon synapse formation and visual function (Regus-Leidig et al., 2014; Muller et

65 al., 2019) as well as the integrity of hippocampal synapses has been identified (Waites et al.,

66 2013). What remains unclear is how Piccolo contributes to cerebellar development and whether,

67 as suggested by genetic studies, it has a primary role in the etiology of PCH3. The recent

68 generation of a Piccolo knockout rat $\left(\mathrm{Pclo}^{g t / g t}\right)$ using transposon mutagenesis (Medrano et al.,

$692019)$ provides an opportunity to explore this potential relationship.

70 In the current study, we have assessed the contribution of Piccolo to cerebellar structure

71 and function through the anatomical, functional and behavioral characterization of adult $P c l o^{g t / g t}$

72 rats. Our analysis reveals a striking number of similarities to patients with $\mathrm{PCH}$, including a

73 smaller cerebral cortex, a reduced volume of the cerebellum and pons as well as impaired motor

74 control and the presence of seizures. Our analysis has also uncovered changes in the anatomical

75 and ultrastructural features of mossy fiber terminals and electrophysiological properties of these

76 synapses. Together, these phenotypes are predicted to not only alter the functionality of the

77 cerebellum but to contribute to motor and perhaps also behavioral dysfunctions seen in PCH3

78 affected individuals. 


\section{Results}

\section{Brain morphology is changed in Piccolo knockout brains.}

81 The recent analysis of a pair of boys with PCH3 identified a non-sense mutation in the coding

82 region of the human Pclo gene (chr7:82579280 G>A), predicted to eliminate the C-terminal third 83 of the longest Piccolo isoforms (Ahmed et al., 2015) and likely its expression. These individuals

84 have profound cognitive and motor impairment as well as atrophy of the cerebrum, cerebellum

85 and pons (Ahmed et al., 2015). A fundamental question is whether Piccolo loss of function is

86 causal for this disorder. To explore this possibility, we have characterized a recently generated

87 line of rats $\left(\mathrm{Pclo}^{g t / g t}\right)$ wherein transposon mutagenesis was used to disrupt the Piccolo gene via an

88 insertion into exon 3 (Figure 1A). This insertion is predicted to cause a frame shift in the reading

89 frame and thus disrupt the expression of full-length Piccolo (560kDa) and most of its alternatively

90 spliced lower molecular weight isoforms (70-350kDa). Schematic is adapted from Ackermann et

91 al. (Ackermann et al., 2019; Medrano et al., 2019). Just after birth (P0-P2) Piccolo pups were

92 found born in normal Mendelian numbers (Figure 1B). Western blot analysis of brain lysates

93 from postnatal day 0-2 (P0-P2) pups demonstrates the loss of nearly all isoforms in homozygous

94 knockout animals (Figure 1C). To assess whether Piccolo loss of function adversely affects brain

95 development, we performed an anatomical characterization of $P c l o^{w t / w t}$ and Pclo ${ }^{g t / g t}$ animals;

$96 \mathrm{Pclo}^{g t / g t}$ pups were smaller and weighed significantly less than their Pclo ${ }^{w t / w t}$ littermates (Figure

97 1D, E and F). However, brain weights were not significantly altered between $P c l o^{g t / g t}$ and $P c l o o^{w t / w t}$

98 littermates at P0-2 and display similar brain morphology (Figure 1G, H and I), suggesting that

99 changes in brain size develop postnatally. However, the overall size and weight of Pclo ${ }^{\text {gt/gt }}$ brains

100 was significantly reduced in 3 month-old adult rats compared to $P c l o^{w t / w t}$ brains (Figure $1 \mathrm{~J}$ and

$101 \mathrm{~K})$. To assess whether this was associated with an overall loss in brain volume or due to

102 reductions in specific brain regions, serial sagittal and coronal sections from 3 month-old animals

103 were collected and stained with Nissl to visualize brain morphology (Figure 1L). Qualitative

104 analysis revealed that thalamic, cerebellar and brainstem regions are dramatically reduced in size 
105 in 3 month-old Pclo gt/gt animals. Some thinning of the cerebral cortex can also be observed,

106 however, no obvious changes in the hippocampus are detectable. Furthermore, ventricles (V1,

$107 \mathrm{~V} 2, \mathrm{~V} 3$ and V4) in $P c l o^{g t / g t}$ brains were all notably larger than in $P c l o^{w t / w t}$ littermates (Figure 1L).

108 Intriguingly, the observed morphological changes in brains of 3 month-old Pclo ${ }^{g t / g t}$

109 animals are remarkably similar to those reported for patients with PCH3, who exhibit

110 microcephaly, a reduced size of the cerebrum, cerebellum and pons as well as larger ventricles

111 (Maricich et al., 2011). As hypoplasia of the pons and cerebellum as well as a reduced thickness

112 of the cortex are the most dramatic features of $\mathrm{PCH} 3$ patients, we examined in more detail

113 changes occurring in these regions of $P c l o^{g t g t}$ brains. Here, we found that the thickness of the

114 cortex is significantly reduced in brains lacking Piccolo (Figure 2A and D). The area of the pons

115 is dramatically reduced in $P c l o^{g t / g t}$ brain slices stained with Nissl (Figure 2B). As the density of

116 neurons was not changed (Figure 2B, zoom), these data indicate a loss of the total number of

117 neurons within the pons. This conclusion is supported by data from brainstem sections stained

118 with antibodies against the synaptic vesicle (SV) protein VGluT1, prominently expressed in pons

119 neurons, which reveals a smaller area occupied by these neurons (Figure 2C and E).

120 Analysis of sagittal sections through the cerebellum of adult rats reveals that, whilst the

121 cerebellum is smaller in Pclo ${ }^{g t g t}$ animals, the overall anatomy is not altered (Figure 3A). For

122 example, there are no remarkable defects in foliation of $P c l o^{g t / g t}$ animals, with all lobes present

123 and appearing to be formed normally at the vermis (Figure 3A and E). Our analysis of the granule

124 cell layer (GCL), using DAPI to stain granule cell (GC) nuclei, reveals that this layer is

125 significantly reduced in size in $P c l o{ }^{g t / g t}$ compared to Pclo ${ }^{w t w t}$ controls (Figure 3A and C).

126 However, this decrease was not associated with a proportional increase in GC packing density, as

127 the number of GCs per GCL area is only very slightly increased (Figure 3B and D). These data

128 indicate an overall loss of GCs in Pclo ${ }^{g t / g t}$ cerebella. Conceptually, this is predicted to reduce the

129 total number of GC parallel fibers innervating PC dendrites in the molecular layer (ML), a 
130 situation that could lead to a thinner ML and perhaps an altered packing density of Purkinje cells 131 (PCs).

132 Sections immunostained with antibodies against Calbindin, which specifically labels PCs,

133 reveals that the organization of the ML and the PC layer (PCL) appear to be intact and that the

134 dendritic arbors of PCs are correctly orientated (Figure 3E, F and I). However, quantifying the

135 average area per unit length of the ML reveals a dramatic reduction in the size of this layer in

$136 P c l o^{g t / g t}$ brains (Figure 3G). Furthermore, cells in the PCL appear overcrowded in Pclo ${ }^{g t / g t}$

137 cerebella (Figure 3E, F and I), a conclusion supported by data showing an increased packing

138 density of PCs in Pclo ${ }^{g t / g t}$ brains (Figure 3H).

139 Taken together, these data indicate a reduced number of GCs and a higher packing 140 density of PCs in Pclo ${ }^{g t / g t}$ cerebella compared to Pclo ${ }^{w t / w t}$ controls.

\section{Loss of Piccolo alters climbing fiber and mossy fiber innervation in the cerebellum.}

143 Anatomically, the cerebellum receives its major excitatory afferents in the form of

144 climbing fibers (CFs) and mossy fibers (MFs) that project from the inferior olive or the

145 brainstem/spinal cord, respectively (Leto et al., 2016). Both form glutamatergic synapses but

146 terminate in different layers of the cerebellum (Apps and Garwicz, 2005). For example, CFs form

147 excitatory synapses onto the proximal branches of PC dendritic arbors, modulating the dynamic

148 firing properties of PCs and motor learning (Hashimoto and Kano, 1998). In contrast, MFs

149 terminate on the dendrites of GCs, which then provide direct excitatory input to PCs via their

150 parallel fiber (PF) axons. Both also extend collaterals to the deep cerebellar nuclei before

151 projecting into the cerebellar cortex (Shinoda et al., 1992).

152 In previous studies, we observed that Piccolo was present in the boutons of each of these

153 excitatory synapses (Cases-Langhoff et al., 1996). This was confirmed by immunostaining

154 cerebellar sections of $\mathrm{Pclo}^{\text {wt/wt }}$ and $\mathrm{Pclo}^{\mathrm{gt} / \mathrm{gt}}$ with antibodies against Piccolo and the SV protein

155 VGlut1 (Fig. 4A and B). This revealed that Piccolo immuno-reactivity was indeed present at each 
156 of these synaptic types and that this immuno-reactivity was lost in cerebella from Pclo ${ }^{g t / g t}$

157 animals. To explore whether deficiencies in either could contribute to the anatomical and

158 functional changes in the cerebellum, we initially analyzed potential differences in the CF input

159 into the ML. Synaptic input from CFs onto PC dendrites, immuno-positive for Calbindin, was

160 visualized with antibodies against VGluT2 (Miyazaki et al., 2003). Here our analysis of sagittal

161 sections revealed the presence of a large number of VGluT2 positive puncta decorating Calbindin

162 positive dendrites in $P c l o o^{g t / g t}$ and $P c l o o^{w t / w t}$ sections (Figure $4 \mathrm{C}$ and D). These data indicate that the

163 loss of Piccolo does not affect the ability of CFs to project into the ML and form synapses with

164 PC soma and dendrites. Qualitatively, VGluT2 positive puncta in Pclo ${ }^{w t / w t}$ and Pclo ${ }^{g t / g t}$ sections

165 were of similar size and beautifully decorated both primary and tertiary PC dendrites, though the

166 total number of puncta appeared more numerous in $P c l o^{g t / g t}$ sections. Quantification of the total

167 area of VGluT2 per ML supports this impression (Figure 4D). Additionally, we compared the

168 distribution of the CFs to the synaptic inputs from GCs onto PCs, the parallel fiber (PF) axons

169 which project into the ML. The latter synapses were identified with antibodies against VGluT1

170 (Miyazaki et al., 2003). Although the ML is thinner in $P c l o^{g t / g t}$ cerebella (Figure 3A), we

171 observed no overt changes in the intensity or distribution of VGluT1 positive puncta throughout

172 the ML of Pclo ${ }^{g t / g t}$ animals compared to Pclo ${ }^{w t / w t}$ controls (Figure 4C). This implies that PF axons

173 project normally and form a robust number of synapses with PCs. Given that fewer GCs are

174 formed in Pclo $^{g t g t}$ cerebella (Figure 3A and B), we postulate that the thinner ML is most likely

175 due to fewer PFs and less total synaptic input on PC dendrites.

176 GCs are known to receive their excitatory input from MFs arising from afferent axons

177 from a number of distinct nuclei in the brainstem including the pontine nuclei (Sillitoe, 2012).

178 These collaterals form large glomerular structures with multiple AZs, forming a rosette of

179 synapses with claws from dendrites of multiple GCs (Jakab and Hamori, 1988; Xu-Friedman and

180 Regehr, 2003). Given the smaller size of the pons, we thus explored whether the boutons from the

181 remaining cells properly reached the cerebellum and formed robust MF terminals. As afferent 
182 fibers from the pons primarily innervate cerebellar lobes VI to IX, we examined sagittal sections

183 of these lobes stained with antibodies against the somatodendritic marker MAP2 and VGluT2 in

184 lobe VII. In $P c l o^{w t / w t}$ sections, multiple large VGluT2 positive puncta are seen packed tightly

185 together within a dense meshwork of MAP2 positive dendrites projecting from a ring of GCs.

186 These puncta represent subclusters of SVs with large 100-200 $\mu \mathrm{m}^{3}$ terminals (Jakab and Hamori,

187 1988). In sections from $P c l o^{g t / g t}$ animals, each bouton appeared smaller in size and less organized,

188 though they still appear to contact GC dendrites (Figure 5A). This impression is further supported

189 by sections immunostained with antibodies against VGluT1 and VGluT2. In these images,

190 distinct VGluT1 and/or VGluT2 positive clusters are observed in both $P c l o^{w t / w t}$ and $P c l o^{g t / g t}$

191 animals, consistent with individual boutons or glomeruli (Figure 5B). Here again, MF terminals

192 from Pclo $^{\text {gt/gt }}$ animals appeared to have much smaller VGluT1 or VGluT2 positive clusters

193 (Figure 5B, zoom). Quantifying the average size of the VGluT1 and VGluT2 positive clusters

194 revealed that clusters of $P c l o{ }^{w t / w t}$ cerebella were more than double the size compared to the same

195 lobes in Pclo $^{g t / g t}$ animals (Figure 5B and C). However, this effect was not lobe-specific as it was

196 observed in all lobes and not just in those receiving pontocerebellar afferents (Figure 5B). These

197 findings suggest that defects in the formation of large robust MF glomeruli in Pclo ${ }^{g t / g t}$ cerebella is

198 a common feature shared by MF afferents arising from the pons and other brainstem nuclei, and

199 may reflect aberrant signaling during development between GCs and these neurons.

200 Quantitatively, the spread of distribution of VGluT1 and VGluT2 cluster sizes is far more

201 shifted towards smaller cluster sizes in $P c l{ }^{g t / g t}$, with mutant cerebellar displaying approximately

$20245 \%$ more small synaptic clusters of $5 \mu^{2}$ in size than $P c l o{ }^{w t w t}$ counterparts (Figure 5D). At the

203 larger end of the scale, cluster sizes over $50 \mu^{2}$ were much more frequent for $P c l o^{w t / w t}$ than $204 P c l o^{g t / g t}$ cerebella (Figure 5E).

205 In addition to excitatory input, MF glomeruli are also modulated by GABAergic

206 inhibition via cerebellar Golgi cells, which offer regulatory feedback to the complex, as they

207 themselves are excited by GCs (Maex and De Schutter, 1998). In principle, smaller MFs 
208 terminals seen in Piccolo KO animals could represent less excitatory input into GCs. This may 209 also reduce excitatory drive onto PCs via PFs from GCs, as well as perhaps inhibitory drive via 210 the Golgi cells. One of the dominant receptors mediating inhibitory input from Golgi cells to GCs 211 are the $\alpha 6$ subunit-expressing $\mathrm{GABA}_{\mathrm{A}}$ receptors, which are highly concentrated within $\mathrm{MF}$ 212 glomeruli (Nusser et al., 1996). Sagittal sections immunostained with antibodies against VGluT2 213 and $\alpha 6$ subunits revealed that the $\mathrm{GABA}_{\mathrm{A}} \alpha 6$ receptor subunit is only weakly expressed in $P c l o^{g t / g t}$ 214 MF rosettes, whereas in Pclo ${ }^{w t w t}$, it nicely localizes to the synaptic complex (Figure 6A).

215 Antibody staining of the granule cell layer demonstrates higher intensity of $\mathrm{GABA}_{\mathrm{A}} \alpha 6$ antibody 216 staining in $P c l o^{w t w t}$ compared to $P c l o^{g t / g t}$ (Figure 6B). However, knockout models of GABA ${ }_{\mathrm{A}} \alpha 6$

217 (Homanics et al., 1997) do not display PCH3 phenotypes or alterations in the anatomy of the 218 cerebellum. Therefore, $\mathrm{GABA}_{\mathrm{A}} \alpha 6$ downregulation can be attributed to Piccolo loss and not for 219 the $\mathrm{PCH} 3$ phenotypes.

These data suggest that the loss of Piccolo not only affects the size of MF inputs into the 221 cerebellum, but also gene expression and therefore inhibitory drive within each glomerulus, a 222 condition that could relate to the maturation of these structures and/or their functionality, a 223 situation that could adversely affect cerebellar function.

225 Ultrastructural analysis of mossy fiber glomeruli in $\boldsymbol{P c l o}^{g t g t}$ cerebellum.

226 The reduced area of VGluT1 and VGluT2 clusters within Pclo ${ }^{g t / g t}$ MF terminals (Figure 227 6) could be due to a reduction in size of MF glomeruli themselves and/or in the number of 228 VGluT1/2 positive SVs per bouton. To explore these options, we investigated MF glomeruli in $229 P c l o^{w t / w t}$ and $P c l o^{g t / g t}$ cerebella using electron microscopy (EM). Analysis of ultrathin cerebellar 230 brain sections from 3 month-old rats revealed that the average size of $P c l o^{g t / g t}$ glomeruli was 231 significantly smaller than Pclo ${ }^{w t / w t}$ glomeruli (Figure 7A and C). Furthermore, the complexity of 232 the glomeruli indicated by a P2A value, measuring the ratio of perimeter per area, was 233 significantly reduced (Figure 7E). The size of the presynaptic area was also reduced in Pclo ${ }^{g t / g t}$ 
234 cerebellar sections (Figure 7B and D). However, the number of active zones (AZs) present at 235 each glomerulus was still proportional to their size, as $P c l o{ }^{w t / w t}$ and $P c l o o^{g t / g t}$ had a similar number 236 of AZs per glomerular area (Figure 7B and F). Given that Pclogtgt boutons are smaller, this 237 indicates that the overall output of the MF glomeruli could be reduced in Pclo ${ }^{\text {gt/gt }}$ cerebellum. 238 Intriguingly, we also noticed an accumulation of clathrin-coated vesicles (CCVs) in the terminals 239 of Pclo $^{\text {gt/gt }}$ MFs (Figure 7G). This phenotype resembles recent findings from hippocampal 240 synapses from $\mathrm{Pclo}^{\mathrm{gt} / \mathrm{gt}}$ animals, which revealed defects in the formation of endosomal membranes

241 and an overall reduction in SV number (Ackermann et al., 2019) and suggest possible changes in 242 the recycling of SVs within MF boutons post fusion with the plasma membrane.

\section{Piccolo loss alters GC properties and mossy fiber to GC synaptic transmission}

245 The anatomical and morphological changes observed in MF boutons lacking Piccolo are 246 predicted to not only represent altered afferent input into the cerebellum from the pons and other 247 brainstem nuclei, but also altered cerebellar function. As an initial test of this hypothesis, we 248 performed whole-cell current clamp recordings of cerebellar GCs from acute P90 rat cerebellar 249 slices. A two-photon image of a typical cerebellar GC from $P c l^{g t / g t}$ filled with ATTO dye reveals 250 a normal radial arrangement of its dendrites as they project their claws into MF glomeruli (Figure $2518 \mathrm{~A}$ ). An analysis of the intrinsic biophysical properties of these cells revealed that the GC 252 properties differed between $P c l o^{g t / g t}$ and $P c l o^{w t / w t}$ animals. Specifically, no changes were detected 253 in either the capacitance or the membrane potential of these cells, but the input resistance was 254 significantly increased (Figure 8B). Since these experiments were performed in the presence of $255 \mathrm{GABA}_{\mathrm{A}}$ receptor blockers, the decreased shunting inhibition mediated by tonic activation of $\alpha 6-$ 256 subunit-containing $\mathrm{GABA}_{\mathrm{A}}$ receptors (Brickley et al., 1996; Nusser et al., 1998) is expected to 257 further increase the difference in input resistance. Yet, there was also no change in the amplitude, 258 threshold of activation or duration of action potentials fired by these cells (Figure 8B), indicating 259 unaltered active membrane properties. Examining the frequency and amplitude of spontaneous 
260 miniature excitatory postsynaptic currents (mEPSCs) of these GCs revealed a dramatic increase

261 in the frequency of these events in $\mathrm{Pclo}^{g t / g t}$ slices with no change in mEPSC amplitudes (Figure

262 8C). These data suggest that on average each excitatory synapse formed on to these GC dendrites

263 has normal levels of postsynaptic AMPA-type glutamate receptors. The change in frequency

264 could either be due to an increase in the number of MF boutons contacting GC dendrites and/or

265 an increase in the release probability of MF boutons. Consistently, the average amplitude of the

266 evoked excitatory postsynaptic currents (EPSCs) was increased in Pclo ${ }^{g t / g t}$ animals, with no

267 change in the weighted time constant $\left(\tau_{\mathrm{w}}\right)$ (Figure 8D). Given the amplitudes of the mEPSCs are

268 not changed, these results suggest that there is a higher number of synaptic connections between

269 MF boutons and the dendrites of GCs, consistent with the larger number of smaller SV

270 clusters/rosette in Pclo ${ }^{g t / g t}$ animals (Figure 6). Taken together, these data indicate that, in addition

271 to changes in cell number, the loss of Piccolo in the cerebellum and brainstem is associated with

272 changes in the morphology and function of GCs and their mossy fiber input.

273

274 Behavioral and motor defects in Piccolo knockout rats.

275 The motor difficulties reported (Zelnik et al., 1996; Durmaz et al., 2009) in humans with

$276 \mathrm{PCH} 3$ and the anatomical changes observed in the cerebellum and brainstem of rats lacking

277 Piccolo predict altered motor function in these animals. To test this hypothesis, Pclo ${ }^{w t / w t}, P_{c l o}{ }^{w t / g t}$

278 and $P c l o^{g t / g t}$ rats were monitored for their motor abilities. In rotarod tasks, Pclo ${ }^{g t / g t}$ rat

279 performance was significantly reduced compared to $P c l o^{w t / w t}$ and $P c l o^{w t / g t}$ rats (Figure 9A).

280 Specifically, while $P c l o^{w t / w t}$ and $P c l o^{w t / g t}$ rats exhibited increasing performance levels regarding

281 the ability to stay on the rotarod over time, clo $^{g t / g t}$ rats showed no indication of being able to

282 adapt to the task. Pclo ${ }^{g t / g t}$ rats were less adept at staying on the task apparatus once rod rotation

283 was initiated (Figure 9A). Intriguingly, no differences in forelimb grip strength were scored

284 between Piccolo genotypes (Figure 9B), indicating that $P c l o^{g t / g t}$ rats showed lack of motivation

285 and/or impaired coordination. 
In addition to deficits in motor ability, $P c l o^{g t / g t}$ rats displayed an increased frequency in

287 front and rear foot stepping errors during ladder rung walking compared to Pclo ${ }^{w t / w t}$ and Pclo ${ }^{w t / g t}$

288 rats (Figure 9C). In open field tests, no significant differences were recorded between Pclo ${ }^{w t / w t}$,

289 Pclo $^{\text {wt/gt }}$ and $P c l o^{g t g t}$ rats for peripheral line crossing or self-grooming events (Figure 9D).

290 However, Pclo ${ }^{g t / g t}$ rats displayed 2- to 3-fold decreases in peripheral rearing events compared to

$291 P c l o^{w t / w t}$ and Pclo ${ }^{w t / g t}$ animals (Figure 9D). Deficits in $P c l o^{g t / g t}$ rat performance during rotarod,

292 ladder rung and open field tests (Figure 9A-D) reflected recessive traits, consistent with

293 dysfunction in proprioceptive sensation and motor control (Curzon et al., 2009).

294 Alongside traditional tests for motor coordination, we tested the behavior of Pclo ${ }^{w t / w t}$,

$295 P c l o^{w t g t}$ and $P c l o^{g t / g t}$ rats in a home cage setup, the OptiMan (Operator Independent Motor-

296 analysis) system, where animals can be monitored without interference from experimenters. Rats

297 were tagged with a radio frequency identification chip that allowed for tracking of locomotor

298 activity while they were also required to complete an isometric pull task that requires precise and

299 finely controlled movements. In the home cage, the $P c l{ }^{g t / g t}$ rats were more active and covered

300 about twice the distance than $P c l o^{w t / w t}$ rats (Figure 9E) during each measurement. In the isometric

301 pull task, performance of $P c l o^{g t / g t}$ rats was significantly lower than the performance of Pclowt/wt

302 rats, quantified using four different force thresholds (Figure 9F). Taken together, Pclo ${ }^{g t / g t}$ rats

303 show clear motor deficits, very similar to symptoms seen in PCH3 patients (Rajab et al., 2003).

\section{Discussion}

306 Our study demonstrates that Piccolo LOF causes alterations in brain anatomy. In

307 particular, the cerebrum, pons, brainstem and cerebellum are severely reduced in size whereas the

308 ventricles are increased (Figure 1). These changes are associated with reductions in cerebellar and

309 pontine cell numbers and perturbations in cerebellar CF and MF afferents (Figure 4 and 5). These

310 changes are predicted to adversely affect cerebellar function - supported by changes in synaptic

311 transmission and motor control in $\mathrm{Pclo}^{\mathrm{gt} / \mathrm{gt}}$ rats. Interestingly, the changes in brain morphology 
312 resemble changes in children with $\mathrm{PCH}$, recently linked to a SNP in the PCLO gene (Ahmed et 313 al., 2015). In addition to brain atrophy, children with PCH3 have, amongst other symptoms, 314 cognitive and motor deficits as well as seizures. Given that seizures were also observed in $P c l o^{g t / g t}$ 315 rats (Medrano et al., 2019), we postulate that $P c l o^{g t / g t}$ rats represent a model to study the 316 underlying mechanisms of this devastating disease.

317 Similar to patients with $\mathrm{PCH} 3, \mathrm{Pclo}^{g t / g t}$ rats have smaller cerebella, brainstem and pontine 318 nuclei. In the cerebellum, a striking change was the thickness of the ML and CGL (Figure 3). The 319 latter was associated with fewer total GCs, which could result in fewer PFs and a thinner ML.

320 The orientation of PCs and the ramification of their dendritic arbors were largely unchanged. At 321 present, it is unclear why there are fewer GCs in $P c l^{g t / g t}$ cerebella. During normal development, 322 several factors including Sonic hedgehog (Wallace, 1999; Miyashita et al., 2017) and Notch 323 (Solecki et al., 2001) are known to control the proliferation of GCs. How Piccolo loss influences 324 these and related signaling pathways is unclear; though Piccolo and Bassoon have been shown to 325 regulate the activity-dependent translocation of c-terminal binding protein 1 (CTPB1) to the 326 nucleus and thereby the expression of neuronal genes (Ivana et al., 2015). CTPB1 and CTPB2 are 327 highly expressed in the cerebellum and their function and/or localization could be affected by 328 Piccolo LOF (Hubler et al., 2012; Ivanova et al., 2015). There is a clear rationale for exploring 329 this phenotype further.

330 In the ML, CF morphology and arrangement appear to be normal, though a higher 331 innervation of PC dendrites by CFs can be observed (Figure 4). One possible explanation for this 332 hyper-innervation is that homeostatic changes to the network, such as heterosynaptic competition 333 with PFs for PC dendrite territory (Hashimoto et al., 2009; Ichikawa et al., 2016) could contribute 334 to these differences in CF distribution and therefore the functionality of the cerebellum. This 335 could affect normal pruning mechanisms of CF during development.

336 Our study also revealed that the pontine nuclei was dramatically decreased in size in $337 P c l o^{g t / g t}$ animals. This is note-worthy as afferent MFs from the pons and other brainstem nuclei 
338 are the primary excitatory input onto GCs, forming elaborate rosette synapses (Voogd and

339 Glickstein, 1998). The smaller size of the pons suggests a net reduction in MF input into the

340 cerebellum that appears to correspond to the reduced number of GCs (Figure 3). However, it is

341 unclear how the loss of Piccolo could influence the number of neurons in the pons.

342 Surprisingly, our histological studies revealed that MF boutons across all lobes of the

343 cerebellum were reduced in size (Figure 5). This finding was supported by our EM studies

344 revealing that MF glomeruli are severely reduced in size, potentially resulting in smaller SV

345 clusters (Figure 7). These observations suggest that the development/maturation of MFs from the

346 pons and other brainstem nuclei are muted in $P c l o^{g t / g t}$ animals. Additionally, electrophysiological

347 changes (Figure 8) indicate that the network has triggered compensatory changes to overcome a

348 smaller size or impaired strength of MFs reaching the GC layer, for instance by increasing both

349 input resistance and release probability (Turrigiano, 2012).

350 Our electron micrographs also showed what could be disturbed synaptic integrity in

$351 P c l o^{g t / g t}$ MF boutons with more CCVs being present (Figure 7), suggesting that the loss of Piccolo

352 may also affect SV recycling. These observations are in accordance with recent findings by

353 Ackermann et al. (2019) of hippocampal synapses from Pclo ${ }^{g t / g t}$ animals. Here, it was reported

354 that the loss of Piccolo had a dramatic effect on the recycling of SV proteins through a functional

355 block in the formation of early endosomes from endocytic vesicles, due to defects in the

356 activation of Rab5 via a Piccolo-dependent loss of Pra1 from synapses. Although beyond the

357 current study, it is likely that this endocytic defect in the recycling of SV proteins could also

358 contribute to altered size function of MF synapses, especially given their high frequency

359 transmission and therefore need for high SV turnover (Byczkowicz et al., 2018).

360 GCs also receive inhibitory modulation from Golgi cells (Eccles et al., 1966) and it is

361 well appreciated that $\mathrm{GABA}_{\mathrm{A}}$ receptors at the MF synapse contain $\alpha 6$ subunits (Nusser et al.,

362 1996). Intriguingly, $P c o^{g t / g t}$ rats display a reduced expression of the $\mathrm{GABA}_{\mathrm{A}} \alpha 6$ subunit at MF

363 rosettes (Figure 6). This is in line with findings from Medrano et al. (2019), who performed RNA 
364 sequencing on the $P c l o^{g t / g t}$ rats and found a severe reduction in $\mathrm{GABA}_{\mathrm{A}} \alpha 6$ subunit gene 365 expression.

366 At present, it is unclear why levels of these subunits are reduced. One possibility is that it 367 reflects a homeostatic change within cerebellar circuitry to compensate for a reduced excitatory 368 input from MFs (Figure 5). This concept is supported by electrophysiological data showing that 369 the input resistance of GCs is higher as is mEPSC frequency and EPSC amplitudes (Figure 8).

370 This condition might arise to compensate for the smaller MF terminals reaching the GCL, which 371 could initially have weaker output properties. Alternatively, reduced $\mathrm{GABA}_{\mathrm{A}} \alpha 6$ expression could 372 be due to lower levels of BDNF, which is secreted from precerebellar neurons, at MF terminals 373 (Chen et al., 2016) and is necessary to promote the formation of GABAergic synapses onto GCs. 374 Though a role of Piccolo in the secretion of BDNF has not been investigated, the expression of 375 Bassoon, which shares significant functional redundancy with Piccolo, is linked to presynaptic 376 levels of BDNF (Heyden et al., 2011). In this regard, the remaining Bassoon protein could 377 suppress BDNF secretion in MF terminals, altering the maturation of the glomeruli and $378 \mathrm{GABA}_{\mathrm{A}} \alpha 6$ expression.

379 An important question not addressed by our studies is why MF boutons are smaller. By 380 several measures mentioned above, it would appear that the MF glomeruli are less mature. Key 381 regulators of MF maturation are members of the Wnt family, a group of target-derived factors 382 which accelerate neuronal maturation or directly induce synapse formation (Scheiffele, 2003; 383 Waites et al., 2005). Interestingly, mouse cerebellar MFs lacking Wnt7a show a similar reduction 384 in MF size and complexity, as we observed in Pclo $^{\text {gt/gt }}$ (Hall et al., 2000). However, it is important 385 to note that in the case of Wnt7a loss, MF synapse size catches up to WT size by age P15, which 386 points to a lag in maturation and does not quite seem to be the case in our $P c l o^{g t g t}$ rats. Knockout 387 of Disheveled1 (Dvl1), a downstream target of Wnt (Salinas and Zou, 2008) also shows a 388 reduction in MF cluster size, indicating that presynaptic Dvl1 is a necessary step in the Wnt 389 signaling cascade, underscoring the importance of these proteins for MF-GC synapse formation 
390 (Ahmad-Annuar et al., 2006). Like Dvl1, Piccolo is located at the AZ of the presynaptic terminal 391 and regulates F-actin assembly and synaptic transmission through its interaction with Daam1 and 392 Profilin (Wagh et al., 2015). Daam1 is a formin and a known regulator/interaction partner of Dvl1 393 (Gao and Chen, 2010). It is thus possible that, in the absence of Piccolo, Dvl1 is not properly 394 localized to presynaptic sites, preventing proper Wnt signaling and consequently causing the 395 formation of smaller and less organized MF-GC synapses. Furthermore, analysis of Pclo ${ }^{g t / g t}$ 396 transcripts reveals that Wnt expression is reduced (Medrano et al., 2019). Thus, there appears to 397 be at least two possible mechanisms that could contribute to smaller MF boutons in Piccolo 398 knockout rats: defects in SV recycling and Wnt signaling. Clearly further studies are needed to 399 explore these options.

400 A fundamental question raised by the anatomical, morphological and functional changes 401 within the cerebellum in rats lacking Piccolo is whether these changes affect the functionality of 402 the cerebellum. In behavioral tests, we observed that $P c l o^{g t g t}$ rats performed significantly worse 403 than both $P c l o^{w t / w t}$ and Pclo ${ }^{w t / g t}$ littermates at motor function tasks, highlighting the recessive 404 nature of the behavioral impairments. Our colleagues Medrano et al. (2019) also observed 405 epileptic seizures and increased aggression in $P c l^{g t / g t}$ rats, and a failure to reproduce due to 406 impaired brain-gonad signaling.

407 Taken together, these data support the concept that Piccolo loss of function in patients 408 with $\mathrm{PCH} 3$ could be causal for many of the observed phenotypes, including changes in the 409 volume of brain structures and behavioral abnormalities such as impaired motor control and 410 epileptic seizures (Rajab et al., 2003; Namavar et al., 2011). With regard to reduced cerebellar 411 function, our studies highlight a prominent role for MF boutons which are not only smaller in size 412 but with altered synaptic properties. Mechanistic studies, which probe how Piccolo loss 413 contributes to these changes, should provide insights into the etiology of this devastating disease.

\section{Materials and Methods}




\section{Generation of Piccolo KO rats $\left(\right.$ Pclo $\left.^{g t / g t}\right)$}

417 Generation of mutant Piccolo rat strains: Mutant rat strains harbored Sleeping Beauty $\beta$-Geo trap

418 transposons (Ivics et al., 2009), originally transmitted from a donor, recombinant rat

419 spermatogonial stem cell library (Izsvak et al., 2010). Recipient males were bred with wildtype

420 females to produce a random panel of mutant rat strains enriched with gene traps in protein

421 coding genes (Izsvak et al., 2010). All experiments were approved by the Institutional Animal

422 Care and Use Committee (IACUC) at UT-Southwestern Medical Center in Dallas, as certified by

423 the Association for Assessment and Accreditation of Laboratory Animal Care International

424 (AALAC) NIH OLAW Assurance \# D16-00296.

\section{Characterization of pups and genotyping}

427 All procedures for experiments involving animals, were approved by the animal welfare 428 committee of Charité Medical University and the Berlin state government. P0-P2 rats were 429 weighed using Kern 440-43N scales and measured for approximate length with a ruler.

430 Genotyping of pups' cortices later revealed their genetic identity.

431 P0-P2 pups were genotyped using a PCR based reaction. In brief, brain tissue was 432 digested in lysis buffer $(100 \mathrm{mM}$ Tris- $\mathrm{HCl}(\mathrm{pH} 8.0)$ with $10 \mathrm{mg} / \mathrm{ml}$ proteinase $\mathrm{K}, 100 \mathrm{mM} \mathrm{NaCl})$

433 for 5 minutes at $55{ }^{\circ} \mathrm{C}$, before inhibiting Proteinase $\mathrm{K}$ by incubation at $99{ }^{\circ} \mathrm{C}$ for 10 minutes.

434 Samples were then centrifuged at $14,800 \mathrm{rpm}$ for 2 minutes and $1 \mu \mathrm{l}$ of supernatant was used for 435 the PCR reaction as outlined below.

436 For determination of genotype for adult rats, earpieces were taken and digested overnight 437 at $55{ }^{\circ} \mathrm{C}$ in SNET-buffer (400 mM NaCl, $1 \%$ SDS, $200 \mathrm{mM}$ Tris (pH 8.0), $5 \mathrm{mM}$ EDTA) 438 containing $10 \mathrm{mg} / \mathrm{ml}$ proteinase $\mathrm{K}$. Proteinase $\mathrm{K}$ enzyme reaction was stopped incubating the 439 samples for $10 \mathrm{~min}$ at $99^{\circ} \mathrm{C}$. The mixture was centrifuged for $2 \mathrm{~min}$ at $14,800 \mathrm{rpm}$. Supernatant 440 was transferred into a fresh tube and DNA was precipitated by addition of $100 \%$ isopropanol.

441 Following samples were centrifuged for $15 \mathrm{~min}$ at $4{ }^{\circ} \mathrm{C}, 13,000 \mathrm{rpm}$. Precipitated DNA was 
442 washed once with $70 \%$ ethanol and centrifuged again for $5 \mathrm{~min}$ at 13,000 rpm. Supernatant was

443 discarded and the DNA pellet was air dried and resuspended in H2O. A PCR reaction with a

444 specific primer combination was performed on isolated DNA. The following primers were used:

445 Pclo KO F2: 3` gcaggaacacaaaccaacaa 5`; Pclo KO R1: 3` tgacctttagccggaactgt 5`; SBF2: 3`

446 tcatcaaggaaacctggac 5 . The PCR reaction protocol was the following: 2 min $94{ }^{\circ} \mathrm{C} ; 3 \times(30 \mathrm{sec}$

$\left.44794{ }^{\circ} \mathrm{C}, 60{ }^{\circ} \mathrm{C} 30 \mathrm{sec}, 72{ }^{\circ} \mathrm{C} 30 \mathrm{sec}\right) ; 35 \mathrm{x}\left(94{ }^{\circ} \mathrm{C} 30 \mathrm{sec}, 55^{\circ} \mathrm{C} 30 \mathrm{sec}, 72{ }^{\circ} \mathrm{C} 30 \mathrm{sec}\right) ; 72{ }^{\circ} \mathrm{C} 10$

448 min. Samples were mixed with a loading dye (New England BioLabs, MA, USA) and run on $2 \%$

449 agarose gel (Serva, Heidelberg, Germany) at $110 \mathrm{~V}$ for $45 \mathrm{~min}$. The gel was imaged using

450 BioDocAnalyze UV transilluminator and BioDocAnalyze2.2 software.

$452 \quad$ Western blot analysis

453 Brains from P0 - P2 pups were lysed in Lysis buffer (50 mM Tris-HCl, $150 \mathrm{mM} \mathrm{NaCl}, 5 \mathrm{mM}$

454 EDTA, $1 \%$ Triton X-100, $0.5 \%$ Deoxycholate, protease inhibitor $\mathrm{pH} 7.5$ ) and incubated on ice

455 for $5 \mathrm{~min}$. Samples were centrifuged at $13,000 \mathrm{rpm}$ for $10 \mathrm{~min}$ at $4{ }^{\circ} \mathrm{C}$. Afterwards the

456 supernatant was transferred into a fresh tube and the protein concentration was determined using

457 a BCA protein assay kit (Thermo Fisher scientific, Waltham, Massachusetts, USA). The same

458 protein amounts for $P c l o^{w t / w t}, P c l o^{w t / g t}$ and $\underline{P c l o^{g t / g t}}$ samples were separated by SDS-PAGE and

459 transferred onto nitrocellulose membranes (running buffer: $25 \mathrm{mM}$ Tris, $190 \mathrm{mM}$ Glycine, $0.1 \%$

460 SDS, pH 8.3; transfer buffer: 25 mM Tris, $192 \mathrm{mM}$ Glycine, $1 \%$ SDS, $10 \%$ Methanol for small

461 proteins, $7 \%$ Methanol for larger proteins $\mathrm{pH}$ 8.3). After the transfer, nitrocellulose membranes

462 were blocked with $5 \%$ milk in TBST (20 mM Tris pH 7.5, $150 \mathrm{mM} \mathrm{NaCl}, 0.1 \%$ Tween 20 ) and

463 incubated with primary antibodies in $3 \%$ milk in TBST overnight at $4{ }^{\circ} \mathrm{C}$. The following

464 antibodies were used: Piccolo (1:1000; rabbit; Synaptic Systems, Göttingen, Germany; Cat\#

465 142002), $\mathrm{GABA}_{\mathrm{A}} \alpha 6$ receptor (1:300; Merck Darmstadt, Germany; Cat\# 06-868) overnight.

466 Nitrocellulose membranes were washed 3 times for 10 min with TBST and incubated with HRP-

467 conjugated secondary antibodies for $1 \mathrm{~h}$ at RT (1:1000; Sigma-Aldrich, St. Louis, USA; Cat\# 
468 NA9310 Cat\# NA934). Membranes were washed 3 times for 10 min with TBST, afterwards

469 secondary antibody binding was detected with ECL Western Blotting Detection Reagents

470 (Thermo Fisher Scientific, Waltham, USA) and a Fusion FX7 image and analytics system (Vilber

471 Lourmat, Collégien, France).

472

473 Immunohistochemistry

474 Immunohistochemistry was performed on brain tissue from rats perfused with $4 \%$

475 paraformaldehyde (Roth, Karlsruhe, Germany) dissolved in PB (80 mM Na2HPO4 (Roth,

476 Karlsruhe, Germany), 20 mM NaH2PO4 (Bernd Kraft, Duisburg, Germany)) (PFA). Brains were

477 extracted and placed in $4 \%$ PFA overnight, cryoprotected in $15 \%$ and then $30 \%$ sucrose

478 solution (Sigma-Aldrich, St. Louis, USA; dissolved in PB) for 24 hours each. Brains were snap-

479 frozen by submersion in 2-methylbutane (Roth, Karlsruhe, Germany) cooled to $-60{ }^{\circ} \mathrm{C}$ and then

480 stored at $-20{ }^{\circ} \mathrm{C}$ until use. Brains were cut para-sagittally or coronally with a Leica cryostat to

481 either $20 \mu \mathrm{m}$ thick sections and mounted on superfrost slides (Thermo Fisher Scientific,

482 Waltham, USA), or $50 \mu \mathrm{m}$ sections which were processed free-floating. Slides were left to dry for

483 a minimum of one hour before storage at $-20{ }^{\circ} \mathrm{C}$ and free-float sections were stored in antifreeze

484 solution (30 \% Ethylene glycol, $30 \%$ Glycerol (Roth, Karlsruhe, Germany), $30 \%$ ddH2O, $10 \%$

485 0.244M PO4 buffer (NaOH, NaH2PO4, Roth, Karlsruhe, Germany)).

486 Prior to staining at least 4 slides (each containing two sections) from each animal were

487 left to equilibrate at room temperature (RT) for one hour. Sections were selected to encompass

488 the range of the axis we were investigating. A hydrophobic barrier was created around sections

489 using a DAKO pen (DAKO, Glostrup, Denmark) and sections were washed and permeabilized

490 with TBS (20 mM Tris pH 7.5, $150 \mathrm{mM} \mathrm{NaCl}$, Roth, Karlsruhe, Germany) with $0.025 \%$ Triton

491 X-100 (Roth, Karlsruhe, Germany) (TBST) for 3 x 5 min, prior to blocking with $10 \%$ Normal

492 Goat Serum (NGS, Sigma-Aldrich, St. Louis, USA) with $1 \%$ Bovine Serum Albumin (BSA,

493 Sigma-Aldrich, St. Louis, USA) in TBS. The following primary antibodies were used: Calbindin 
494 (1:750; rabbit; Synaptic Systems, Göttingen, Germany; Cat\# 214002), GABA receptor $\alpha$ subunit 495 (1:250; rabbit; Sigma-Aldrich, St. Louis, USA; Cat\# G5544), MAP2 (1:1000; chicken; 496 MilliporeSigma, Burlington, USA, Cat\# AB5543), Piccolo (1:200; guinea pig; Synaptic Systems, 497 Göttingen, Germany; Cat\# 142 104), VGluT1 (1:1000; rabbit; Abcam, Cambridge, UK; Cat\# 498 ab104898), VGluT2 (1:250; guinea pig; Synaptic Systems, Göttingen, Germany; Cat\# 499 13540419). VGluT2 (1:300; mouse monoclonal (epitope AA 566 to 582); Synaptic Systems, 500 Göttingen, Germany; Cat\# 135 421). Antibodies diluted in TBS with $1 \%$ BSA were applied and 501 left overnight at $4{ }^{\circ} \mathrm{C}$. After 3 x 5 min washing with TBST, differently labeled secondary 502 antibodies were used from Invitrogen (Thermo Fisher Scientific, Waltham, USA, dilution 503 1:1000), again diluted in $1 \%$ BSA in TBS antibody solution and then applied for 1 hour at RT.

504 Sections were then washed with TBS $2 \times 10$ min or, if desired, incubated in TBS with DAPI

505 (Roth, Karlsruhe, Germany) for $30 \mathrm{~min}$ before washing again 2 x $10 \mathrm{~min}$. Slides were 506 coverslipped (24x50 mm coverslips, Menzel Gläser, Braunschweig, Germany) with Immu-Mount 507 (Shandon-Thermo Scientific, Cheshire, UK) and sealed with clear nail polish once hardened.

$509 \quad$ Nissl stain

510 Sections were washed 3 times in PBS then mounted onto superfrost slides and allowed to dry for 511 1-2 days. Slides were inserted into slide racks and passed through the following solutions: $95 \%$ 512 EtOH (Ethanol from Roth, Karlsruhe, Germany; diluted as appropriate with ddH2O) x 15 min, 70 $513 \%$ EtOH, $50 \%$ EtOH, ddH2O, 10 min Blue counterstain (TACS from Trevigen, MD, USA), $514 \mathrm{ddH} 2 \mathrm{O}$ and then dehydrated through $50 \%, 70 \%$ acid EtOH (1 \% glacial acetic acid (Th. Geyer, 515 Renningen, Germany) in $70 \%$ EtOH), $95 \%$ and $100 \%$ EtOH before clearing in Roti Histol 516 (Roth, Karlsruhe, Germany), coverslipped using Entellan mounting medium (Entellan, 517 Darmstadt, Germany) and left to dry for 24 hours under a fume hood.

519 Confocal z-stack image acquisition and processing 
520 Images were acquired on a spinning disc confocal microscope (Zeiss Axio Observer.Z1 with

521 Andor spinning disc and cobolt, omricron, i-beam laser) using a 40x (1.3 NA) and 100x (1.4 NA)

522 Plan-Apochromat oil objective and an iXon ultra (Andor, Belfast, UK) camera controlled by iQ

523 software (Andor, Belfast, UK). Sections for $\mathrm{GABA}_{\mathrm{A}} \alpha 6$ analysis (Figure 7) were imaged with a

524 Nikon Spinning Disk Confocal CSU-X using a 100x (1.45 NA) Plan Apo oil objective and an

525 EMCCD camera with Andor Revolution SD System(Andor, Belfast, UK).

526

527 Tile scan overview images

528 An upright microscope (Olympus BX61) was used to image fluorescently stained cerebellar

529 sections. A CCD color camera was used with a 10x or 5x lens for overview pictures. CellSens

530 software (Olympus, Hamburg, Germany) stitched multiple images together to give an overview

531 of the whole cerebellar region.

532

533 Image analysis

534 For image processing and analysis ImageJ/FIJI software was used (Schindelin et al., 2012). For

535 analysis of $P c l o{ }^{w t / w t}$ and $P c l o{ }^{g t / g t}$ tissue sections were selected from the vermis and approximately

536 every 10th slide laterally (each slide containing 2 sections) was analyzed. The best quality section

537 per slide was imaged. For layer thickness, all lobes were measured and for closer analysis of MF

538 size, CF coverage and GC density, images were taken from lobes I, III, V, VII and IX where

539 possible. For $\mathrm{GABA}_{a} \mathrm{a} 6$ subunit expression, 6 images were taken per slide, 4 slides per animal.

540 Average signal intensity was measured for the whole field of view in the GABAa6 antibody

541 channel and then normalized to the MAP2 channel for each image.

542 Layer 'thickness' was calculated per lobe by dividing the area of the layer by the inner

543 length of the layer for both GCL and ML. For Purkinje cell density, the number of PCs per lobe

544 was counted and divided by the length of the PC layer for that lobe. Data points represent

545 'thickness' values from individual lobes. 
546 Granule cell density was calculated from 100x magnification single plane images stained

547 with DAPI. GCs were counted per image by an experimenter blinded to the genotype of each

548 image, and this number divided by the area of each image. Data points represent each image. .

549 Mossy fiber cluster size was measured using a script within ImageJ to analyze particles

550 stained with VGluT1 and/or VGluT2. The area of each puncta was measured and an average

551 taken per image. Data points represent average cluster size of each image.

552 Climbing fiber innervation was assessed using VGluT2 and Calbindin staining. The

553 molecular layer area was defined in the Calbindin channel, consisting of Calbindin-positive PC

554 dendrites, and the VGluT2 channel was selected and pasted into a new imageJ file. The same

555 script as for MF analysis was run to calculate the total area stained with VGluT2, divided by the

556 area of the ML and x100 to give percentage coverage. Data presented represent percentage per

557 image.

558

559 Electron microscopy

$560 P c l o o^{w t / w t}$ and $P c l o{ }^{g t / g t}$ rats were anesthetized deeply with $33 \mathrm{mg} / \mathrm{ml}$ Ketamine (Inresa Arzneimittel

$561 \mathrm{GmbH}$, Freiburg, Germany) $830 \mu \mathrm{g} / \mathrm{ml}$ Xylavet (cp-pharma, Burgdorf, Germany) and perfused

562 transcardially with $37^{\circ} \mathrm{C}$ physiological saline for 3 to 4 min followed by a $0.1 \mathrm{M}$ phosphate

563 buffer containing $4 \%$ paraformaldehyde and $0.05 \%$ glutaraldehyde. Brains were stored in

564 fixative overnight and subsequently sliced sagittally $(350 \mu \mathrm{m})$ on a vibratome. Regions of interest

565 were cut into small pieces and post-fixed in $1 \% \mathrm{OsO} 4$ and $0.1 \mathrm{M}$ sodium cacodylate and en-bloc

566 stained in $1 \%$ uranyl acetate aqueous solution. Finally, samples were dehydrated and embedded

567 in epoxy resin (Epon 812; EMS). Ultrathin sections were cut using an Ultracut ultramicrotome

568 (UCT, Leica, Wetzlar, Germany) equipped with a diamond knife (Ultra 45, DiATOME, Hatfield,

569 USA) and collected on formvar-coated 200-mesh copper grids (EMS). Sections were imaged in a

570 Zeiss EM-900 Transmission Electron Microscope (Zeiss) operated at $80 \mathrm{keV}$ and equipped with a

$5712 \mathrm{~K} \times 2 \mathrm{~K}$ CCD camera. Data were analyzed blindly using the ImageJ software. Data points 
572 represent analysis from individual images obtained from 3 rats per genotype. For histological and

573 EM data, a normality test was performed (D'Agostino-Pearson omnibus normality test). If

574 successful then a student's t-test was used to compare $P c l o^{w t / w t}$ to $P c l o^{g t / g t}$ rats, or alternatively a

575 test for non-normal data (Mann-Whitney U test) was used.

576

577 Electrophysiology methods

578 Cerebellar slices were prepared from 3-to-4 month-old Pclowtwt and Pclo ${ }^{g t / g t}$ rats of either sex.

579 Animals were treated in accordance with the German Protection of Animals Act and with the

580 guidelines for the welfare of experimental animals issued by the European Communities Council;

581 local authorities approved the experiments. Animals were anesthetized with isoflurane (Baxter,

582 Deerfield, IL) followed by a rapid decapitation with a custom-built guillotine. The cerebellar

583 vermis was quickly removed and mounted in a chamber filled with chilled extracellular solution.

584 Parasagittal $300 \mu \mathrm{m}$ slices were cut using a Leica VT1200 microtome (Leica Microsystems,

585 Wetzlar, Germany), transferred to an incubation chamber at $\sim 35^{\circ} \mathrm{C}$ for 30 min and subsequently

586 stored at RT. Artificial cerebrospinal fluid (ACSF) was used for slice cutting, storage, and

587 experiments. ACSF contained: $125 \mathrm{mM} \mathrm{NaCl}, 25 \mathrm{mM} \mathrm{NaHCO}, 2.5 \mathrm{mM} \mathrm{KCl}, 1.25 \mathrm{mM}$

$588 \mathrm{NaH} 2 \mathrm{PO} 4,1.1 \mathrm{mM} \mathrm{CaCl} 2,1 \mathrm{mM} \mathrm{MgCl} 2,3 \mathrm{mM}$ Glucose, $17 \mathrm{mM}$ Sucrose $(\sim 310 \mathrm{mOsm}, \mathrm{pH} 7.3$

589 when bubbled with Carbogen (5\% O2/95\% CO2)). Patch pipettes were pulled from borosilicate

590 glass (Science Products, Hofheim, Germany) using a DMZ Puller (Zeitz-Instruments,

591 Martinsried, Germany). Patch pipettes had open-tip resistances of 6-9 M $\Omega$. The intracellular

592 solution contained: $150 \mathrm{mM}$ K-gluconate, $10 \mathrm{mM} \mathrm{NaCl}, 10 \mathrm{mM}$ K-HEPES, 3 mM Mg-ATP, 0.3

$593 \mathrm{mM} \mathrm{Na-GTP}$ (300-305 mOsm, pH adjusted to 7.3 with $\mathrm{KOH})$. In some of the experiments, the

594 intracellular solution contained $10 \mu \mathrm{M}$ of the fluorescence dye Atto594. Experiments were

595 performed at $35-37{ }^{\circ} \mathrm{C}$ and slices were continuously superfused with ACSF containing $20 \mu \mathrm{M}$

596 SR95531 and $40 \mu \mathrm{M}$ D-(2R)-amino-5-phosphonovaleric acid (D-APV) to block Golgi-cell 
597 inhibition and NMDA-receptors, respectively. Atto594 was obtained from Atto-Tec (Atto-Tec,

598 Siegen, Germany); all other chemicals were purchased from Sigma-Aldrich (St. Louis, MO).

\section{Current clamp recordings.}

601 To determine the input resistance, subthreshold current pulses were applied from -20 to $+20 \mathrm{pA}$

602 in 2pA steps. Action potentials were evoked in current-clamp mode by current pulses (amplitude

$60320-400 \mathrm{pA}$, duration $300 \mathrm{~ms}$ ). The resistance of the solution-filled patch-pipettes was $24.9 \pm 1$

$604 \mathrm{M} \Omega$ and $23 \pm 1 \mathrm{M} \Omega$ for $P c l o o^{w t / w t}$ and $P c l o{ }^{g t / g t}$ rats respectively. Patch-clamp recordings were made

605 using a HEKA EPC10/2 USB amplifier (HEKA Elektronik, Lambrecht/Pfalz, Germany). Data

606 were sampled at $200 \mathrm{kHz}$. Measurements were corrected for a liquid junction potential of +16

$607 \mathrm{mV}$.

608 Excitatory postsynaptic currents (EPSCs)

609 To measure evoked EPSCs, GCs were held at a holding potential of - $80 \mathrm{mV}$ and Mossy fiber

610 axons were stimulated at $1 \mathrm{~Hz}$. The stimulation voltage ranged between 16 to $40 \mathrm{~V}$ for control

611 and Pclo ${ }^{g t / g t}$ animals. For spontaneous EPSCs GCs were held at $-80 \mathrm{mV}$ for around 3 minutes.

612 Single events were detected using the Igor Pro extension NeuroMatic (Rothman and Silver, 613 2018b) tool for Event detection.

614 The current clamp data were analyzed using custom-made procedures in Igor Pro 615 software (WaveMetrics, Oregon, USA) as described previously (PMID:31379501). In short, 616 properties of action potentials of GCs were determined from the injected currents, that elicited the 617 largest number of action potentials (APs). The action potential threshold was defined as the 618 membrane voltage at which the first derivative exceeded $100 \mathrm{~V} \mathrm{~s}^{-1}$, the minimal AP peak was set 619 as $-20 \mathrm{mV}$ and the minimal amplitude to $20 \mathrm{mV}$. All APs with a half-width smaller than $50 \mu \mathrm{s}$ 620 and higher than $500 \mu$ s were excluded. AP frequency and AP half-width were calculated from the

621 first three APs. Membrane capacitance, resting membrane potential and series resistance were 622 read from the amplifier software (HEKA) after achieving the whole-cell configuration. Input 
623 resistance $\left(\mathrm{R}_{\text {in }}\right)$ was analyzed by plotting the steady-state voltage elicited by the subthreshold

624 current injections against the injected current and a spline interpolation was performed to obtain

625 the slope at the holding membrane potential ( $0 \mathrm{pA}$ current injection).

626

\section{Excitatory postsynaptic currents}

628 To measure evoked EPSCs, GCs were held at a holding potential of - $80 \mathrm{mV}$ and mossy fiber

629 axons were stimulated extracellular with a second patch pipette at $1 \mathrm{~Hz}$. For spontaneous EPSCs

$630 \mathrm{GCs}$, were held at $-80 \mathrm{mV}$ for around 3 minutes. Single events were detected using the template

631 detection algorithm of the Igor Pro extension NeuroMatic (Rothman and Silver, 2018a).

632

633 EPSCs were analyzed with the Igor Pro software. The amplitude and the kinetics were

634 determined from the average of 25 individual single EPSCs. To obtain the decay kinetics, single

635 EPSCs were fitted with one or two exponentials. The weighted time constant was calculated as:

636

$$
\tau w=\frac{\text { Aslow } \tau \text { slow }+ \text { Afast } \tau \text { fast }}{\text { Aslow }+ \text { Afast }}
$$

638 Behavioral assessment

$639 \underline{\text { Rotarod task }}$

640 The apparatus (IITC Life Science, Woodland Hills, CA) consisted of 5 semi-enclosed lanes and

641 an elevated metal rod $(9.525 \mathrm{~cm}$ diameter, $29.21 \mathrm{~cm}$ elevation) with a fine textured finish to

642 enhance grip. For each trial, all rats were placed on the unmoving rod, allowed to stabilize their

643 posture, and then rod rotation was initiated. Test parameters were: rotation direction, toward

644 investigator to encourage rats to face away while walking; start speed, $4 \mathrm{rpm}$; top speed, $44 \mathrm{rpm}$;

645 acceleration rate, $0.2 \mathrm{rpm} / \mathrm{s}$ (200 sec from start to top speed); max test duration, $300 \mathrm{~s}$. Each rat's

646 trial ended when it fell from the rod, triggering the fall-detection sensor. Data was automatically 
647 recorded to a computer. Rats underwent 4 trials/day, with an inter-trial interval of at least $10 \mathrm{~min}$

648 for 4 consecutive days.

649

$650 \quad$ Ladder rung task

651 Ladder rung tests were performed on cohorts of Pclo ${ }^{w t / w t}$, Pclo $^{\text {wt/gt }}$ and Pclo ${ }^{g t / g t}$ rats by methods

652 previously described (Metz and Whishaw, 2009). Rats were trained to cross a plexiglass tunnel

653 about $1 \mathrm{~m}$ long with metal rods provided at regular intervals as steps. Each step that the rat took

654 was scored on the basis of paw placement on a 7 category scale with 0 being paw totally missing

655 the rung and 6 being correct paw placement. The average score per pair of limbs was used to

656 quantify step score. During the trial, the error per step was also quantified by number of low

657 scoring steps (0-2) divided by the total number of steps that the rat took during the test with each

658 pair of limbs (student's t-test). Data were derived from 3 trials with $3 \mathrm{~cm}$ rung separation

659 conducted on the same test day with at least a $10 \mathrm{~min}$ inter-trial interval, and group means were

660 compared using one-way ANOVA and Bonferroni post-hoc tests.

661

662 Grip test

663 To assess grip strength, rats were allowed to cling on to a support by forelimbs or hindlimbs and

664 pulled (Curzon et al., 2009). The support is attached to a transducer that can measure the pull

665 force being applied on the rat by the tester. During each trial, force that was necessary to be

666 applied by the tester to release the grip of the rat was recorded. Three trials per limb pair were

667 done and the means compared by student's t-test. To assess grip strength, the rat's torso was

668 supported ventrally while both forelimbs were allowed to grasp a metal support bar, then the rat

669 was pulled in a horizontal plane until the bar was released. Peak force was measured by a

670 transducer attached to the support bar (San Diego Instruments, San Diego, USA). Nine trials per

671 rat were conducted over two days (inter-trial interval at least $5 \mathrm{~min}$ ) and group means were

672 compared using one-way ANOVA and Bonferroni post-hoc tests. 


\section{Open field test}

675 The open field test (Curzon et al., 2009) was performed for each rat using a rectangular arena 676 (91.44 $\mathrm{cm} \times 60.96 \mathrm{~cm})$ divided into 9 equal sectors (1 center, 8 perimeter). At the start of the 677 experiment, the rat was placed in the center sector and allowed to explore for $10 \mathrm{~min}$. The test 678 was recorded by digital video for later analysis. One test was conducted per rat. The behaviors

679 counted were crossing, rearing and grooming. Each crossing event was counted when all four 680 limbs of the test subject crossed the boundary of one sector into another. Rearing was defined as 681 the number of times the subject stood up on its hind limbs. Grooming was defined as the number 682 of times a rat licked or scratched itself while remaining stationary. Events that occurred in the 683 center versus perimeter sectors were tallied separately, and group means were analyzed for each 684 region by one-way ANOVA and Bonferroni post-hoc tests.

687 Experiments were approved by the animal welfare committee of Charité Medical University and

688 the Berlin state government. Four groups (22 male \& 4 female) of Pclo ${ }^{w t / w t}$, Pclo $^{\mathrm{wt} / g t}$ and $P c l o^{g t / g t}$ 689 rats were used in this study. Rats carried subcutaneous radio-frequency identification (RFID) tags 690 in ventral location. Animals were group housed with 6 - 8 rats per group. Initially, all rats were 691 habituated to the OptiMan multi-cage environment with open sorter gates for voluntary 692 exploration. Then, automated sorting was activated so that only one rat could enter the operant 693 chamber at a time. During pull-task training, force thresholds and handle positions were adapted 694 every day for each session to the current skill level reached by a rat. Each session had 20 trials, 695 and two to three individual sessions were given per day with an intersession interval of 30 to 60 696 min. Within a session, the maximum time interval a rat was allowed to remain inactive between 697 trials was $6-8 \mathrm{~min}$. A session ended when such inactivity occurred. The OptiMan (Operator 698 Independent Motor-Analysis, PhenoSys) system consists of two interconnected group home cages 
699 (EU Type IV cage and 2000P) resting on an RFID sensor array plate that automatically tracks

700 RFID tag movement within the cage, and thus the locomotor activity of individual rats, day and

701 night. One of the home cages was connected via an electronic guillotine gate to a second cage

702 resting on a balance that automatically determined the body mass of a rat when inside this cage.

703 Individual animals voluntarily and self-motivated decided to visit the balance cage. Subsequent to

704 the balance cage, and again separated by an electronic guillotine gate, was a cage compartment

705 containing a horizontal ladder $(1 \mathrm{~m})$ with electronically monitored dual rungs on the left and right

706 side. From this ladder compartment, an animal entered a cage containing the isometric pull-task.

707 From there back to the home cage a rat passed over a force grid sensor array that sensed ground

708 forces exerted by the paws.

$710 \quad$ Isometric pull task

711 Rats were trained to pull a handle attached to a stationary force transducer with a predetermined

712 force threshold upon which they received a sugar pellet reward. Upon a rat's entry into the

713 isometric pull task cage a sugar pellet was delivered into the reward tray. A session started when

714 the rat retrieved this first reward, which led to the automatic closing of the entry gate, and to the

715 motorized slide-in appearance of the force handle to its predefined position. During each session,

7165 different handle positions were presented to a rat. These positions varied from $11 \mathrm{~mm}$ inside the

717 cage wall (easiest position), to $7 \mathrm{~mm}$ outside of the cage wall (most difficult position). Handle

718 positions changed automatically during a session through a motorized slide. The difficulty level

719 within each handle position increased stepwise by increasing the force threshold for pellet release.

720 This started at $30 \mathrm{~g}$ pull-force and was increased to $40 \mathrm{~g}, 50 \mathrm{~g}$ and finally $60 \mathrm{~g}$. A trial started

721 when a pull-force of $5 \mathrm{~g}$ was sensed. From then on, the pull-force was sampled for a duration of 4

722 sec. If a rat reached the force threshold within a $2 \mathrm{sec}$ time interval then a trial was successful and

723 a reward delivered. The schedule then advanced to the next level of difficulty by either increasing

724 force threshold or moving the handle one position further towards the outside. Thus, a rat needed 
725 a minimum of 20 trials to complete a session with 5 different handle positions and 4 different

726 force thresholds at each position. If the threshold was not reached then a trial was considered

727 unsuccessful and the rat had to continue with its next trial with unchanged conditions.

728 Experiments lasted for 15 experimental days with 2 - 3 daily sessions per individual.

730 Animal welfare

731 All animals were treated and cared for in accordance with national and institutional guidelines:

732 Generation of mutant Piccolo rat strains and behavioral experiments Figure 10 a-e: Rat

733 protocols were approved by the Institutional Animal Care and Use Committee (IACUC) at UT-

734 Southwestern Medical Center in Dallas, as certified by the Association for Assessment and

735 Accreditation of Laboratory Animal Care International (AALAC), permit number: NIH OLAW

736 Assurance \# D16-00296.

737 Western blotting, immunohistochemistry, electron microscopy and behavioral

738 experiments with Optiman setup (Figure $10 \mathrm{~d}$ and e): Animals were treated in accordance with

739 the German Protection of Animals Act (TierSchG $\S 4$ Abs. 3); all procedures for experiments

740 involving animals were approved by the animal welfare committee of Charite Medical University

741 and the Berlin state government, permit number: T 0036/14.

742 Electrophysiology: Animals were treated in accordance with the German Protection of Animals

743 Act (TierSchG $\S 4$ Abs. 3) and with the guidelines for the welfare of experimental animals issued

744 by the European Communities Council Directive of 24. November 1986 (86/609/EEC). The local

745 authorities approved the experiments (Landesdirektion Leipzig), permit number: T24/18

$747 \quad$ Experimental design and statistical analysis

748 GraphPad Prism (RRID:SCR_002798) was used to analyze and represent data. Statistical design, 749 sample sizes and tests for each experiment can be found in the figure legends. 


\section{References}

752

753

754

755

756

757

758

759

760

761

762

763

764

765

766

767

768

769

770

771

772

773

774

775

776

777

778

779

780

781

782

783

784

785

786

787

788

789

790

791

792

793

794

795

796

797

798

799

800

Ackermann F, Schink KO, Bruns C, Izsvak Z, Hamra FK, Rosenmund C, Garner CC (2019) Critical role for Piccolo in synaptic vesicle retrieval. Elife 8.

Ahmad-Annuar A, Ciani L, Simeonidis I, Herreros J, Fredj NB, Rosso SB, Hall A, Brickley S, Salinas PC (2006) Signaling across the synapse: a role for Wnt and Dishevelled in presynaptic assembly and neurotransmitter release. J Cell Biol 174:127-139.

Ahmed MY, Chioza BA, Rajab A, Schmitz-Abe K, Al-Khayat A, Al-Turki S, Baple EL, Patton MA, Al-Memar AY, Hurles ME, Partlow JN, Hill RS, Evrony GD, Servattalab S, Markianos K, Walsh CA, Crosby AH, Mochida GH (2015) Loss of PCLO function underlies pontocerebellar hypoplasia type III. Neurology 84:1745-1750.

Apps R, Garwicz M (2005) Anatomical and physiological foundations of cerebellar information processing. Nat Rev Neurosci 6:297-311.

Brickley SG, Cull-Candy SG, Farrant M (1996) Development of a tonic form of synaptic inhibition in rat cerebellar granule cells resulting from persistent activation of GABAA receptors. J Physiol 497 ( Pt 3):753-759.

Byczkowicz N, Ritzau-Jost A, Delvendahl I, Hallermann S (2018) How to maintain active zone integrity during high-frequency transmission. Neurosci Res 127:61-69.

Cases-Langhoff C, Voss B, Garner AM, Appeltauer U, Takei K, Kindler S, Veh RW, De Camilli P, Gundelfinger ED, Garner CC (1996) Piccolo, a novel $420 \mathrm{kDa}$ protein associated with the presynaptic cytomatrix. Eur J Cell Biol 69:214-223.

Chen AI, Zang K, Masliah E, Reichardt LF (2016) Glutamatergic axon-derived BDNF controls GABAergic synaptic differentiation in the cerebellum. Sci Rep 6:20201.

Curzon P, Zhang M, Radek RJ, Fox GB (2009) The Behavioral Assessment of Sensorimotor Processes in the Mouse: Acoustic Startle, Sensory Gating, Locomotor Activity, Rotarod, and Beam Walking. In: Methods of Behavior Analysis in Neuroscience (nd, Buccafusco JJ, eds). Boca Raton (FL).

Durmaz B, Wollnik B, Cogulu O, Li Y, Tekgul H, Hazan F, Ozkinay F (2009) Pontocerebellar hypoplasia type III (CLAM): Extended phenotype and novel molecular findings. J Neurol 256:416-419.

Eccles JC, Llinás R, Sasaki K (1966) The mossy fibre-granule cell relay of the cerebellum and its inhibitory control by Golgi cells. Experimental Brain Research 1:82-101.

Fenster SD, Garner CC (2002) Gene structure and genetic localization of the PCLO gene encoding the presynaptic active zone protein Piccolo. Int J Dev Neurosci 20:161-171.

Fenster SD, Chung WJ, Zhai R, Cases-Langhoff C, Voss B, Garner AM, Kaempf U, Kindler S, Gundelfinger ED, Garner CC (2000) Piccolo, a presynaptic zinc finger protein structurally related to bassoon. Neuron 25:203-214.

Gao C, Chen YG (2010) Dishevelled: The hub of Wnt signaling. Cell Signal 22:717-727.

Gundelfinger ED, Reissner C, Garner CC (2015) Role of Bassoon and Piccolo in Assembly and Molecular Organization of the Active Zone. Front Synaptic Neurosci 7:19.

Hall AC, Lucas FR, Salinas PC (2000) Axonal remodeling and synaptic differentiation in the cerebellum is regulated by WNT-7a signaling. Cell 100:525-535.

Hashimoto K, Kano M (1998) Presynaptic origin of paired-pulse depression at climbing fibrePurkinje cell synapses in the rat cerebellum. J Physiol 506 ( Pt 2):391-405.

Hashimoto K, Ichikawa R, Kitamura K, Watanabe M, Kano M (2009) Translocation of a "winner" climbing fiber to the Purkinje cell dendrite and subsequent elimination of "losers" from the soma in developing cerebellum. Neuron 63:106-118.

Heyden A, Ionescu MCS, Romorini S, Kracht B, Ghiglieri V, Calabresi P, Seidenbecher C, Angenstein F, Gundelfinger ED (2011) Hippocampal enlargement in Bassoon-mutant mice is associated with enhanced neurogenesis, reduced apoptosis, and abnormal BDNF levels. Cell Tissue Res 346:11-26. 
801

802

803

804

805

806

807

808

809

810

811

812

813

814

815

816

817

818

819

820

821

822

823

824

825

826

827

828

829

830

831

832

833

834

835

836

837

838

839

840

841

842

843

844

845

846

847

848

849

850

Homanics GE, Ferguson C, Quinlan JJ, Daggett J, Snyder K, Lagenaur C, Mi Z-P, Wang X-H, Grayson DR, Firestone LL (1997) Gene Knockout of the $\alpha 6$ Subunit of the $\gamma$ Aminobutyric Acid Type A Receptor: Lack of Effect on Responses to Ethanol, Pentobarbital, and General Anesthetics. Molecular Pharmacology 51:588-596.

Hubler D, Rankovic M, Richter K, Lazarevic V, Altrock WD, Fischer KD, Gundelfinger ED, Fejtova A (2012) Differential spatial expression and subcellular localization of CtBP family members in rodent brain. PLoS One 7:e39710.

Human Protein Atlas (2015) PCLO. In.

Ichikawa R, Hashimoto K, Miyazaki T, Uchigashima M, Yamasaki M, Aiba A, Kano M, Watanabe M (2016) Territories of heterologous inputs onto Purkinje cell dendrites are segregated by mGluR1-dependent parallel fiber synapse elimination. Proc Natl Acad Sci U S A 113:2282-2287.

Ivanova D, Dirks A, Montenegro-Venegas C, Schone C, Altrock WD, Marini C, Frischknecht R, Schanze D, Zenker M, Gundelfinger ED, Fejtova A (2015) Synaptic activity controls localization and function of CtBP1 via binding to Bassoon and Piccolo. EMBO J 34:1056-1077.

Ivics Z, Li MA, Mates L, Boeke JD, Nagy A, Bradley A, Izsvak Z (2009) Transposon-mediated genome manipulation in vertebrates (vol 6, pg 415, 2009). Nat Methods 6:546-546.

Izsvak Z, Frohlich J, Grabundzija I, Shirley JR, Powell HM, Chapman KM, Ivics Z, Hamra FK (2010) Generating knockout rats by transposon mutagenesis in spermatogonial stem cells. Nat Methods 7:443-445.

Jakab RL, Hamori J (1988) Quantitative Morphology and Synaptology of Cerebellar Glomeruli in the Rat. Anat Embryol 179:81-88.

Leto K et al. (2016) Consensus Paper: Cerebellar Development. Cerebellum 15:789-828.

Maex R, De Schutter E (1998) Synchronization of golgi and granule cell firing in a detailed network model of the cerebellar granule cell layer. J Neurophysiol 80:2521-2537.

Maricich SM, Aqeeb KA, Moayedi Y, Mathes EL, Patel MS, Chitayat D, Lyon G, Leroy JG, Zoghbi HY (2011) Pontocerebellar Hypoplasia: Review of Classification and Genetics, and Exclusion of Several Genes Known to Be Important for Cerebellar Development. Journal of Child Neurology 26:288-294.

Medrano GA, Singh M, Plautz EJ, Good LB, Chapman KM, Chaudhary J, Jaichander P, Powell HM, Pudasaini A, Shelton JM, Richardson JA, Xie X-J, Ivics Z, Braun C, Ackermann F, Garner CC, Izsvák Z, Hamra FK (2019) Mutant screen reveals depression-associated Piccolo's control over brain-gonad cross talk and reproductive behavior. bioRxiv:405985.

Metz GA, Whishaw IQ (2009) The ladder rung walking task: a scoring system and its practical application. J Vis Exp.

Miyashita S, Adachi T, Yamashita M, Sota T, Hoshino M (2017) Dynamics of the cell division orientation of granule cell precursors during cerebellar development. Mech Dev 147:1-7.

Miyazaki T, Fukaya M, Shimizu H, Watanabe M (2003) Subtype switching of vesicular glutamate transporters at parallel fibre-Purkinje cell synapses in developing mouse cerebellum. Eur J Neurosci 17:2563-2572.

Muller TM, Gierke K, Joachimsthaler A, Sticht H, Izsvak Z, Hamra FK, Fejtova A, Ackermann F, Garner CC, Kremers J, Brandstatter JH, Regus-Leidig H (2019) A Multiple PiccolinoRIBEYE Interaction Supports Plate-Shaped Synaptic Ribbons in Retinal Neurons. J Neurosci 39:2606-2619.

Namavar Y et al. (2011) Clinical, neuroradiological and genetic findings in pontocerebellar hypoplasia. Brain 134:143-156.

Nusser Z, Sieghart W, Somogyi P (1998) Segregation of different GABAA receptors to synaptic and extrasynaptic membranes of cerebellar granule cells. J Neurosci 18:1693-1703. 
851

852

853

854

855

856

857

858

859

860

861

862

863

864

865

866

867

868

869

870

871

872

873

874

875

876

877

878

879

880

881

882

883

884

885

886

887

888

889

890

891

892

893

894

895

896

897

898

899

900
Nusser Z, Sieghart W, Stephenson F, Somogyi P (1996) The alpha 6 subunit of the GABAA receptor is concentrated in both inhibitory and excitatory synapses on cerebellar granule cells. The Journal of Neuroscience 16:103-114.

Rajab A, Mochida GH, Hill A, Ganesh V, Bodell A, Riaz A, Grant PE, Shugart YY, Walsh CA (2003) A novel form of pontocerebellar hypoplasia maps to chromosome 7q11-21. Neurology 60:1664-1667.

Regus-Leidig H, Fuchs M, Lohner M, Leist SR, Leal-Ortiz S, Chiodo VA, Hauswirth WW, Garner CC, Brandstatter JH (2014) In vivo knockdown of Piccolino disrupts presynaptic ribbon morphology in mouse photoreceptor synapses. Front Cell Neurosci 8:259.

Rothman JS, Silver RA (2018a) NeuroMatic: An Integrated Open-Source Software Toolkit for Acquisition, Analysis and Simulation of Electrophysiological Data. Front Neuroinform 12:14.

Rothman JS, Silver RA (2018b) NeuroMatic: An Integrated Open-Source Software Toolkit for Acquisition, Analysis and Simulation of Electrophysiological Data. Front Neuroinform 12.

Rudnik-Schoneborn S, Barth PG, Zerres K (2014) Pontocerebellar hypoplasia. Am J Med Genet C Semin Med Genet 166C:173-183.

Salinas PC, Zou YM (2008) Wnt signaling in neural circuit assembly. Annual Review of Neuroscience 31:339-358.

Scheiffele P (2003) Cell-cell signaling during synapse formation in the CNS. Annual Review of Neuroscience 26:485-508.

Schindelin J, Arganda-Carreras I, Frise E, Kaynig V, Longair M, Pietzsch T, Preibisch S, Rueden C, Saalfeld S, Schmid B, Tinevez JY, White DJ, Hartenstein V, Eliceiri K, Tomancak P, Cardona A (2012) Fiji: an open-source platform for biological-image analysis. Nat Methods 9:676-682.

Shinoda Y, Sugiuchi Y, Futami T, Izawa R (1992) Axon Collaterals of Mossy Fibers from the Pontine Nucleus in the Cerebellar Dentate Nucleus. Journal of Neurophysiology 67:547560.

Sillitoe R, Fu, Y., Watson, C. (2012) Chapter 11 - Cerebellum: Academic Press.

Solecki DJ, Liu XL, Tomoda T, Fang Y, Hatten ME (2001) Activated Notch2 signaling inhibits differentiation of cerebellar granule neuron precursors by maintaining proliferation. Neuron 31:557-568.

Turrigiano G (2012) Homeostatic synaptic plasticity: local and global mechanisms for stabilizing neuronal function. Cold Spring Harb Perspect Biol 4:a005736.

Voogd J, Glickstein M (1998) The anatomy of the cerebellum. Trends Neurosci 21:370-375.

Wagh D, Terry-Lorenzo R, Waites CL, Leal-Ortiz SA, Maas C, Reimer RJ, Garner CC (2015) Piccolo Directs Activity Dependent F-Actin Assembly from Presynaptic Active Zones via Daam1. PLoS One 10:e0120093.

Waites CL, Craig AM, Garner CC (2005) Mechanisms of vertebrate synaptogenesis. Annual Review of Neuroscience 28:251-274.

Waites CL, Leal-Ortiz SA, Okerlund N, Dalke H, Fejtova A, Altrock WD, Gundelfinger ED, Garner CC (2013) Bassoon and Piccolo maintain synapse integrity by regulating protein ubiquitination and degradation. EMBO J 32:954-969.

Wallace VA (1999) Purkinje-cell-derived Sonic hedgehog regulates granule neuron precursor cell proliferation in the developing mouse cerebellum. Curr Biol 9:445-448.

Xu-Friedman MA, Regehr WG (2003) Ultrastructural contributions to desensitization at cerebellar mossy fiber to granule cell synapses. Journal of Neuroscience 23:2182-2192.

Zelnik N, Dobyns WB, Forem SL, Kolodny EH (1996) Congenital pontocerebellar atrophy in three patients: clinical, radiologic and etiologic considerations. Neuroradiology 38:684687. 
bioRxiv preprint doi: https://doi.org/10.1101/774422; this version posted September 25, 2019. The copyright holder for this preprint (which was not certified by peer review) is the author/funder. All rights reserved. No reuse allowed without permission.

901 Zhai RG, Vardinon-Friedman H, Cases-Langhoff C, Becker B, Gundelfinger ED, Ziv NE, Garner 902 903 904 CC (2001) Assembling the presynaptic active zone: a characterization of an active one precursor vesicle. Neuron 29:131-143.

905 
908 Figure 1. Generation of $P c{ }^{g t / g t}$ mutant animals.

909 A) Sleeping beauty transposon mutagenesis was used to generate gene trap (gt) piccolo knockout

910 rats. The transposon element inserted into exon 3 of the piccolo genomic sequence and caused a

911 stop in the reading frame. Adapted from Ackermann et al. (Ackermann et al., 2019).

912 B) Pairs of heterozygous $\left(\right.$ Pclo $\left.^{\text {wt/gt }}\right)$ males and females produced litters with Mendelian

913 distribution. Pie chart demonstrates the birth rates of homozygous wildtype (Pclowt ${ }^{w t}$ ),

914 homozygous gene trap mutation $\left(\right.$ Pclo $\left.^{g t g t}\right)$ and heterozygous $\left(P c l o^{w t / g t}\right)$ pups.

915 C) Western blot analysis of brain lysates prepared from postnatal day 2 (P2 ) animals to confirm

916 the loss of full-length Piccolo protein from the brain. A band of the Piccolo-corresponding size of

$917560 \mathrm{kDa}$ is detectable in lysates prepared from $P c l o^{w t / w t}$ and Pclo ${ }^{w t / g t}$ animals, but is absent in

$918 P c l o^{g t / g t}$ brain lysates (data are representative of 3 independent experiments). However, smaller

919 bands between 100 and $70 \mathrm{kDA}$ are still present in $P c l o^{g t / g t}$ brain lysates.

920 D-F) Image of postnatal day 1 (P1) littermates (D), E) Quantification of the body length of P0-P2

$921 P c l o^{w t / w t}$ and $P c l o^{g t / g t}$ pups $\left(P c l o o^{w t / w t}=5.5 \mathrm{~cm} \pm 0.077, \mathrm{n}=23 ; P c l o^{g t / g t}=5.15 \mathrm{~cm} \pm 0.070, \mathrm{n}=27 ; 6\right.$

922 independent litters; unpaired t-test, $\left.p^{* *}=0.0014\right)$. F) Quantification of the body weight of P0-P2

$923 P$ Pclo ${ }^{w t / w t}$ and $P c l o^{g t / g t}$ pups $\left(P c l o{ }^{w t / w t}=8.09 \mathrm{~g} \pm 0.203, \mathrm{n}=39 ; P c l o o^{g t / g t}=7.31 \mathrm{~g} \pm 0.166, \mathrm{n}=35 ; 12\right.$

924 independent litters; unpaired t-test, $\left.p^{* *}=0.0044\right)$.

925 G-H) Image of brains dissected from P1 Pclo ${ }^{w t / w t}$ and Pclo ${ }^{g t / g t}$ pups (G). H) Quantification of the

926 brain weight of P0-P2 Pclo ${ }^{w t / w t}$ and Pclo ${ }^{g t / g t}$ pups $\left(P c l o^{w t / w t}=0.293 \pm 0.00533, \quad \mathrm{n}=7\right.$;

$927 P c l^{g t / g t}=0.289 \mathrm{~g} \pm 0.00758 \mathrm{n}=3 ; 3$ independent litters; unpaired t-test, $p=0.683$ )

928 I) Nissl stained sagittal sections from P2 rat brains show no overt differences between Pclo ${ }^{g t / g t}$ 929 and $P$ clo ${ }^{w t / w t}$ pups. Scale bar $=0.5 \mathrm{~cm}$.

$930 \mathrm{~J}-\mathrm{L}$ ) Image of 4\% PFA-perfused brains from $\mathrm{Pclo}^{w t / w t}$ and $P c l o^{g t / g t}$ animals at 3 months of age (I),

931 K) Quantification of the brain weight showing that $P c o^{g t / g t}$ brains are significantly lighter than 
$932 P$ Pclo ${ }^{w t / w t}$ brains $\left(\right.$ Pclo $^{w t / w t}=2.098 \mathrm{~g} \pm 0.074$, Pclo $^{g t / g t}=1.435 \mathrm{~g} \pm 0.021 ; \mathrm{n}=6$, Mann-Whitney U

933 test, $\left.p^{* *}=0.0022\right)$.

934 L) Nissl stained sagittal sections from 3 month-old rat brains reveal microcephaly in Pclo ${ }^{g t / g t}$

935 compared to $P c l o{ }^{w t / w t}$. Note, ventricles are larger and cerebellum, pons, cerebrum and subcortical

936 regions are smaller.

937 Scale bars: $1 \mathrm{~cm}(\mathrm{G}$ and $\mathrm{K}) ; 0.5 \mathrm{~cm}(\mathrm{I})$. Error bars represent SEM.

938

939 Figure 2. Pclo $^{g t / g t}$ rats show cortical thinning and a smaller pons area compared to $P c l o^{w t / w t}$

940 littermates.

941 A-B) Nissl stained somatosensory cortex (indicated by dashed lines) (A) and the brainstem

942 including pontine area (B) of $\mathrm{Pclo}^{\text {wt/wt }}$ and $\mathrm{Pclo}^{\mathrm{gt} / \mathrm{gt}}$ brains at 3 month of age. B) Zoom

943 demonstrates that pontine neurons are of similar density in $P c l o^{w t w t}$ and $P c l o^{g t / g t}$.

944 C) Pontine area visualized by staining with antibodies against VGluT1 and subsequent DAB

945 conversion of $P c l o^{w t / w t}$ and $P c l o{ }^{g t / g t}$ brains at 3 month of age.

946 D) Quantification of the thickness of the somatosensory cortex $\left(P c l o{ }^{w t / w t}=2.18 \mathrm{~mm} \pm 0.045, \mathrm{n}=\right.$

94725 brain sections; Pclo ${ }^{g t / g t}=1.98 \mathrm{~mm} \pm 0.041, \mathrm{n}=21$ brain sections; $\mathrm{n}=3$ independent

948 experiments; unpaired t-test, $\left.p^{* *}=0.0018\right)$.

949 E) Quantification of the area of the pons $\left(P c l o^{w t w t}=12.24 \mathrm{~mm}^{2} \pm 0.620, \mathrm{n}=26\right.$ brain sections;

$950 P$ Pclo ${ }^{g t / g t}=5.58 \mathrm{~mm}^{2} \pm 0.333, \mathrm{n}=17$ brain sections; $\mathrm{n}=3$ independent experiments; unpaired t-

951 test, $p * * * *<0.0001)$.

952 Scale bars: $200 \mu \mathrm{m}$ (A and C), $500 \mu \mathrm{m}$ (B and C) and $100 \mu \mathrm{m}$ (B, zoom). Error bars represent

953 SEM.

954

955 Figure 3. Morphometric comparison of $\mathrm{Pclo}^{\mathrm{gt} / \mathrm{gt}}$ and $\mathrm{Pclo}{ }^{w t / w t}$ cerebella.

956 A-D) Images of sagittal sections of $P c l o{ }^{w t / w t}$ and $P c l o{ }^{g t / g t}$ cerebella at 3 month of age. The densely

957 packed granule cell layer (GCL) is visualized by DAPI staining. B) Higher magnification images 
958 of (A) demonstrating GC density in the GCL. C) Quantification of the GCL thickness (Pclo ${ }^{w t / w t}=$

$959200.8 \mu \mathrm{m} \pm 2.932, \mathrm{n}=160$ lobes; $P c l o^{g t / g t}=134.8 \mu \mathrm{m} \pm 2.859, \mathrm{n}=148$ lobes; $\mathrm{n}=4$ independent

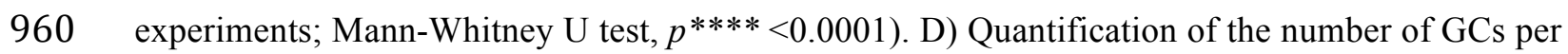

$961100 \mu \mathrm{m}^{2}\left(\right.$ Pclo $^{w t / w t}=1.702 \pm 0.020, \mathrm{n}=111$ images; $P$ clo ${ }^{g t / g t}=1.156 \pm 0.022, \mathrm{n}=114$ images; $\mathrm{n}=$

9623 independent experiments; unpaired t-test, $\left.p^{* *}=0.0042\right)$.

963 E-H) Images of sagittal sections of $P c l o^{w t / w t}$ and $P c l o^{g t g t}$ cerebella at 3 months of age. Purkinje

964 cells (PCs) stained with antibodies against Calbindin determine the molecular layer (ML) (lobes

965 I-III shown). F) Higher magnification images of (E). Note the closer packing of PCs in Pclo ${ }^{g t / g t}$

966 compared to Pclo ${ }^{w t / w t}$. G) Quantification of the ML thickness $\left(P c l o o^{w t / w t}=187.2 \mu \mathrm{m} \pm 2.719, \mathrm{n}=\right.$

967148 lobes; $P$ clo ${ }^{\text {gt/gt }}=127.2 \mu \mathrm{m} \pm 2.378, \mathrm{n}=125$ lobes; $\mathrm{n}=4$ independent experiments; Mann-

968 Whitney U test, $\left.p^{* * * *}<0.0001\right)$. H) Quantification of the number of PCs per $100 \mu \mathrm{m}$ length of

969 PC layer $\left(\right.$ Pclo ${ }^{w t / w t}=1.797 \pm 0.036 ; \mathrm{n}=89$ lobes; Pclo ${ }^{g t / g t}=2.554 \pm 0.058 ; \mathrm{n}=65$ lobes; $\mathrm{n}=3$

970 independent experiments; unpaired t-test, $p * * * *<0.0001)$.

971 I) Images of sagittal sections stained with antibodies against Calbindin showing that PCs migrate

972 correctly to their position in the ML and are correctly orientated.

973 Scale bars: $1 \mathrm{~cm}$ (A), $20 \mu \mathrm{m}$ (B and J), $50 \mu \mathrm{m}$ (I) and $200 \mu \mathrm{m}$ (F). Error bars represent SEM. Data

974 points represent images taken from lobes I, III, V, VII and IX; 4 sections per animal (B and D).

976 Figure 4. Abberant climbing fiber innervation of Purkinje cells in Pclot ${ }^{g t / g t}$ rats compared to $977 \quad$ Pclo ${ }^{w t / w t}$ littermates.

978 A and B) Images of sagittal sections of $P c l o{ }^{w t / w t}$ and $P c l o^{g t / g t}$ cerebella at 3 months of age stained 979 with antibodies against VGluT1 and Piccolo demonstrate the loss of Piccolo in the ML (A) and 980 the GCL (B) in Pclo ${ }^{\text {gt/gt }}$ rats.

981 C) Images of sagittal sections of $P c l o^{w t w t}$ and $P c l o^{g t / g t}$ cerebella at 3 months of age stained with 982 antibodies against Calbindin and VGluT2. Note that the climbing fiber synapses, immuno983 positive for VGluT2, are increased in the ML of $P c l o^{g t / g t}$ cerebella compared to Pclo ${ }^{w t / w t}$ controls 
984 (C and D). When Piccolo is absent, parallel fiber synapses in the ML - immuno-positive for

985 VGluT1 - do not appear overtly different between $P c l o^{w t / w t}$ and $P c l o^{g t / g t}$ (A). However, MF and CF 986 synapses are altered (4B-D, Figure 5).

987 D) Quantification of the percentage of the ML (indicated by dashed lines) immuno-positive for

988 VGluT2 from (C) $\left(\right.$ Pclo $^{w t / w t}=3.521 \pm 0.160, \mathrm{n}=128$ images; $P$ clo ${ }^{g t / g t}=4.377 \pm 0.241, \mathrm{n}=112$

989 images; $\mathrm{n}=3$ independent experiments; Mann-Whitney $\mathrm{U}$ test, $p^{*}=0.0278$ ).

990 Scale bars: $20 \mu \mathrm{m}$. Error bars represent SEM. Data points represent images taken from lobes I,

991 III, V, VII and IX; 4 sections per animal.

992

993 Figure 5. Cerebella from $P c l o^{g t / g t}$ animals have smaller mossy fiber rosettes.

994 A and B) Images of sagittal sections of $P c l o{ }^{w t / w t}$ and $P c l o{ }^{g t / g t}$ cerebella at 3 month of age stained

995 with antibodies against VGluT2, which is highly expressed at mossy fiber (MF) boutons, and the 996 somatodendritic marker MAP2 (A) or VGluT2 and VGluT1 (B). A) GCs extend their dendrites 997 into MF boutons in the Pclo ${ }^{w t / w t}$ condition. However, in $P c l o^{g t / g t}$, whilst GC dendrites are still 998 juxtaposed to VGluT2-positive boutons, the boutons are smaller and therefore the arrangement is 999 less organized. B) Presynaptic MF glomeruli from lobes I (upper panel) and VII (lower panel) are 1000 visualized by VGluT1 and VGluT2. Note that the reduction in MF size is consistent regardless of 1001 the lobe. Note also that rosettes are generally labeled with either VGluT1 or VGluT2 and 1002 occasionally with both markers consistent with them being innervated by a single synaptic input 1003 from different neuronal cell types.

1004 C) Quantification of the size of VGluT1/VGluT2 clusters $\left(P c l o{ }^{w t / w t}=22.73 \mu \mathrm{m}^{2} \pm 0.896, \mathrm{n}=4\right.$ 1005 animals; $P c l o^{g t / g t}=9.46 \mu \mathrm{m}^{2} \pm 0.2899, \mathrm{n}=4$ animals; unpaired t-test, $p^{* * * *<0.0001) .}$

1006 D-E) Histograms to show the distribution of puncta sized 5-50 $\mu \mathrm{m}^{2}$ (D) and $50-260 \mu \mathrm{m}^{2}(\mathrm{E})$. The 1007 shift of the data indicates $P c l o^{g t / g t}$ MFs have more smaller puncta $\left(5 \mu^{2}\right)$, whereas $P c l o{ }^{w t / w t}$ MFs 1008 have more larger puncta (up to $260 \mu \mathrm{m}^{2}$ ). 
1009 Scale bars: $50 \mu \mathrm{m}$ (A and B) and $20 \mu \mathrm{m}$ (B, zoom). Error bars represent SEM. Data points 1010 represent average puncta size per animal from images taken from lobes I, III, V, VII and IX, 4 1011 sections per animal (C, D and E).

1012

1013 Figure 6. GABA $\mathrm{A}_{\mathrm{A}} \alpha 6$ subunit expression is lower in $P c l o^{g t / g t}$ than in $P c l o^{w t / w t}$.

1014 A) Representative images of sagittal sections of $P c l o^{w t / w t}$ and $P c l o{ }^{g t / g t}$ cerebella at 3 months of age

1015 stained with antibodies against the GABA $\mathrm{A}_{\mathrm{A}}$ subunit $\alpha 6$,VGluT2 and MAP2. Note the decreased

1016 levels of $\mathrm{GABA}_{\mathrm{A}} \alpha 6$ in the GC layer of $P c l o^{g t / g t}$ animals compared to $P c l o{ }^{w t / w t}$ controls, quantified 1017 in B).

1018 B) Quantification of $\mathrm{GABA}_{\mathrm{A}}$ subunit $\alpha 6$, measured by the average intensity of antibody staining

1019 in images taken from the GC of the cerebellum, normalized to MAP2 antibody intensities for the

1020 same image $\left(P c l o^{w t / w t}=0.661 \pm 0.0479\right.$ arbitrary units (a.u.), $\mathrm{n}=45$ images from 3 individual

1021 animals; $P$ clo ${ }^{g t / g t}=0.443 \pm 0.26$ a.u., $\mathrm{n}=44$ images from 3 individual animals; Mann-Whitney U

1022 test, $\left.p^{* * *}=0.0009\right)$.

1023 Scale bars: $20 \mu \mathrm{m}$. Error bars represent SEM. Data points represent images taken from 4 sections

1024 per animal.

1025

1026 Figure 7. Glomerular rosettes are smaller and less complex in Pclo ${ }^{g t / g t}$.

1027 A, B and G) Electron microscopy images of the GCL of Pclo ${ }^{w t / w t}$ and Pclo ${ }^{g t / g t}$ cerebella at 3 1028 months of age. Granule cells are indicated by 'gc' (A and B), cerebellar glomeruli are highlighted 1029 in blue $\left(P c l o^{w t / w t}\right)$ and green $\left(P c l o^{g t / g t}\right)(\mathrm{A})$ and the presynaptic terminals of cerebellar MF are 1030 highlighted in blue $\left(P c l o o^{w t / w t}\right)$ and green $\left(P c l o^{g t / g t}\right)(\mathrm{B})$. Asterisks mark active zones (AZs) (B) and 1031 the zoom in $(\mathrm{G})$ emphasizes the presence of more clathrin-coated vesicles (CCVs) in Pclo ${ }^{\text {gt/gt }} \mathrm{MF}$ 1032 boutons compared to than Pclo ${ }^{w t / w t}$.

1033 (C-F) Quantification of the size of glomeruli (C), the size of the MF presynapse (D), the 1034 complexity (squared perimeter/presynaptic area) of the MF bouton (E) and the density of AZs 
1035 (F). Note the strong decrease in glomerulus and MF bouton size (C and D) (C) $\left(P c l o{ }^{w t / w t}=34.59\right.$

$1036 \mu \mathrm{m}^{2} \pm 1.287, \mathrm{n}=130$ images; $P c{ }^{g t / g t}=18.82 \mu \mathrm{m}^{2} \pm 0.853, \mathrm{n}=103$ images; $\mathrm{n}=3$ independent

1037 experiments; Mann-Whitney U test, $p^{* * * *}<0.0001$. (D) $\left(\right.$ Pclo $^{w t / w t}=17.73 \mu \mathrm{m}^{2} \pm 0.603, \mathrm{n}=141$

1038 images; Pclo ${ }^{g t / g t}=11.69 \mu \mathrm{m}^{2} \pm 0.497, \mathrm{n}=95$ images; $\mathrm{n}=3$ independent experiments; Mann-

1039 Whitney U test, $\left.p^{* * * *}<0.0001\right)$. (E) $\left(\right.$ Pclo ${ }^{w t / w t}=90.5 \pm 4.089, \mathrm{n}=141$ images; Pclo ${ }^{g t / g t}=58.1 \pm$

$10403.818, \mathrm{n}=84$ images; $\mathrm{n}=3$ independent experiments; Mann-Whitney $\mathrm{U}$ test, $\left.p^{* * * *}<0.0001\right)$.

1041 (F) $\left(\right.$ Pclo ${ }^{w t / w t}=0.625 \pm 0.021, \mathrm{n}=121$ images; $P$ clo $o^{g t / g t}=0.652 \pm 0.032, \mathrm{n}=84$ images; $\mathrm{n}=3$

1042 independent experiments; Mann-Whitney U test, $p=0.7390$ ).

1043 Scale bars: $2.5 \mu \mathrm{m}(\mathrm{A}), 1 \mu \mathrm{m}(\mathrm{B}), 500 \mathrm{~nm}(\mathrm{G})$ and $250 \mathrm{~nm}(\mathrm{G}$, zoom). Error bars represent SEM.

1044

1045 Figure 8. Physiological assessment of mossy fiber boutons.

1046 A) Example Two-photon image of a cerebellar granule cell (GC) from a Pclogtgt rat filled with

1047 ATTO dye.

1048 B) Average data of biophysical properties of GCs for $P c l o{ }^{w t / w t}$ and $P c l o^{g t / g t}$ rats. The input 1049 resistance of GCs was higher in $P c l o^{g t g t}$ compared to $P c l{ }^{w t / w t}(\operatorname{Pclowt} / \mathrm{wt}=722.6 \mathrm{M} \Omega \pm 76.24, \mathrm{n}$ $1050=22$ cells; $P$ clo ${ }^{g t / g t}=1160 \mathrm{M} \Omega \pm 154.9, \mathrm{n}=23$ cells; $\mathrm{n}=3$ rats per genotype; Mann-Whitney $\mathrm{U}$ 1051 test, $\left.p^{*}=0.0462\right)$. Whereas no differences were found in capacitance $\left(\right.$ Pclo $^{w t w t}=3.717 \mathrm{pF} \pm$ $10520.269, \mathrm{n}=21$ cells; Pclo ${ }^{\text {gt/gt }}=3.643 \mathrm{pF} \pm 0.231, \mathrm{n}=23$ cells; $\mathrm{n}=3$ rats per genotype; Mann1053 Whitney U test, $p=0.912)$, resting membrane potential $(\mathrm{Vm})\left(P c l o^{w t / w t}=-99.96 \mathrm{mV} \pm 1.261, \mathrm{n}=\right.$ 105418 cells, $P$ clo ${ }^{\text {gt } g t}=-100.8 \mathrm{mV} \pm 0.786, \mathrm{n}=23$ cells; $\mathrm{n}=3$ rats per genotype; Mann-Whitney U 1055 test, $p=0.612)$, the half-duration of the action potential $\left(P c l o{ }^{w t / w t}=179.7 \mu \mathrm{s} \pm 11.84, \mathrm{n}=18\right.$ cells; $1056 P$ clo ${ }^{g t / g t}=172.2 \mu \mathrm{s} \pm 7.366, \mathrm{n}=23$ cells; $\mathrm{n}=3$ rats per genotype; Mann-Whitney $\mathrm{U}$ test, $p=$ 1057 0.866), the amplitude of the action potential $\left(P c l o{ }^{w t / w t}=67.85 \mathrm{mV} \pm 3.016, \mathrm{n}=18\right.$ cells; $P$ clo ${ }^{g t / g t}=$ $105863.92 \mathrm{mV} \pm 2.761, \mathrm{n}=23$ cells; $\mathrm{n}=3$ rats per genotype; Mann-Whitney $\mathrm{U}$ test, $p=0.291$ ) and the 1059 voltage threshold to elicit an action potential $\left(P c l o{ }^{w t / w t}=-51.27 \mathrm{mV} \pm 1.748, \mathrm{n}=18\right.$ cells; $P$ clo ${ }^{g t / g t}$ $1060=-48.6 \mathrm{mV} \pm 1.659, \mathrm{n}=23$ cells; $\mathrm{n}=3$ rats per genotype; Mann-Whitney $\mathrm{U}$ test, $p=0.162$ ). 
1061 C) Miniature excitatory postsynaptic currents from Pclo ${ }^{g t / g t}$ GCs were not different in their 1062 amplitude $\left(P c l o^{w t / w t}=-19.62 \mathrm{pA} \pm 1.682, \mathrm{n}=15\right.$ cells; $P$ clo ${ }^{g t / g t}=-22.44 \mathrm{pA} \pm 1.765, \mathrm{n}=23$ cells;

$1063 \mathrm{n}=3$ rats per genotype; Mann-Whitney U test, $p=0.286)$ but in their frequency $\left(P c l o^{w t / w t}=0.102\right.$

$1064 \mathrm{~Hz} \pm 0.0167, \mathrm{n}=15$ cells; Pclo ${ }^{g t / g t}=0.257 \mathrm{~Hz} \pm 0.0481, \mathrm{n}=22$ cells; $\mathrm{n}=3$ rats per genotype;

1065 Mann-Whitney U test, $p^{*}=0.0392$ ).

1066 D) Excitatory postsynaptic currents from GCs measured after stimulation of single mossy fibers 1067 were increased in $P c l o^{g t / g t}$ compared to $P c l o{ }^{w t / w t}\left(P c l o o^{w t / w t}=47.58 \mathrm{pA} \pm 12.12, \mathrm{n}=13\right.$ cells; $1068 P c l o^{g t / g t}=67.62 \mathrm{pA} \pm 9.64, \mathrm{n}=15$ cells; $\mathrm{n}=3$ rats per genotype; Mann-Whitney $\mathrm{U}$ test, $p^{*}=$ 1069 0.0356), whereas the decay of the EPSC was not alerted $\left(P c l o^{w t / w t}=1.79 \mathrm{~ms} \pm 0.258, \mathrm{n}=12\right.$ cells; $1070 P c l o^{g t / g t}=1.404 \mathrm{~ms} \pm 0.141, \mathrm{n}=14$ cells; $\mathrm{n}=3$ rats per genotype; Mann-Whitney test $\mathrm{U}, p^{*}=$ 1071 0.231). Right hand panel: example traces of evoked EPSCs, as quantified in D), in response $1 \mathrm{~Hz}$ 1072 stimulation in the presence of $20 \mu \mathrm{M}$ SR95531 and $40 \mu \mathrm{M} \mathrm{D}-(2 \mathrm{R})$-amino-5-phosphonovaleric 1073 acid (D-APV).

1074 Scale bar $=20 \mu \mathrm{m}(\mathrm{A})$. Error bars represent SEM. Data points represent individual cells from 3 1075 rats per genotype.

1077 Figure 10. Behavioral outcome of Piccolo loss of function resembles PCH3 symptoms.

1078 A) Rotarod performance for $P c l o{ }^{w t / w t}, P c l o o^{w t / g t}$ and $P c l o o^{g t / g t}$ rats for 16 trials over 4 days. Pclo ${ }^{g t / g t}$ 1079 rats fell significantly faster than $P c l o^{w t w t}$ on trials 6 and 8 and faster than both $P c l o o^{w t / w t}$ and $1080 P$ clo ${ }^{w t / g t}$ on trials 9-16 (trial 6: $P$ clo ${ }^{w t / w t}=56.25 \mathrm{~s} \pm 5.023, P c l o o^{g t / g t}=3.5 \pm 0.5, p^{*}=0.0293$; trial 8: $1081 P$ Pclo ${ }^{w t / w t}=65.25 \mathrm{~s} \pm 18.688, P_{c l}{ }^{g t / g t}=4.5 \pm 0.957, p^{* *}=0.0042 ;$ trial 9: $P c l o{ }^{w t / w t}=63.25 \mathrm{~s} \pm$ 108216.163, Pclo $^{w t g t}=61.5 \mathrm{~s} \pm 18.319$, Pclo $^{\text {gt/gt }}=3.25 \pm 0.75, p^{* *}=0.0051, p^{\# \#}=0.0079($ where $*=$ $1083 p$ value between $P c l o^{w t / w}$ and $P c l o^{g t / g t}$ and ${ }^{\#}=p$ value between $P c l o^{w t / w t}$ and $\left.P c l o^{w t / g t}\right)$; trial 10 : $1084 P$ Pclo ${ }^{w t / w t}=84.5 \mathrm{~s} \pm 6.225, P c l o{ }^{w t / g t}=60.25 \mathrm{~s} \pm 18.396, P c l o o^{g t / g t}=2.5 \pm 0.5, p^{* * * *}=<0.0001, p^{\# \#}=$

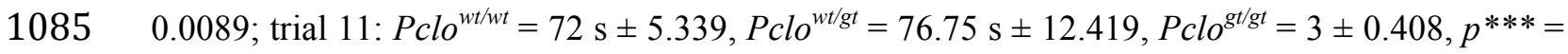

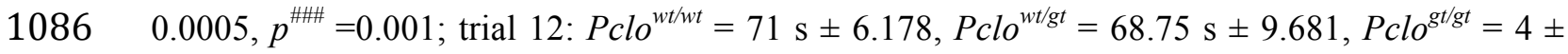


$0.707, p^{* * *}=0.0008, p^{\# \#}=0.0015 ;$ trial 13: clo $^{w t / w t}=59 \mathrm{~s} \pm 8.297$, Pclo $^{w t / g t}=57.75 \mathrm{~s} \pm 12.99$,

$P_{c l o}^{g t / g t}=2.5 \pm 0.5, p^{*}=0.0121, p^{\#}=0.0164 ;$ trial $14: P c l o{ }^{w t / w t}=69.25 \mathrm{~s} \pm 12.479$, Pclo $^{w t / g t}=61 \mathrm{~s}$

$1089 \pm 13.638, P_{c l o}^{g t / g t}=2.5 \pm 0.645, p^{* * *}=0.0009, p^{\# \#}=0.0074 ;$ trial 15: Pclo ${ }^{w t / w t}=60.5 \mathrm{~s} \pm 16.297$,

$1090 P$ Pclo ${ }^{w t / g t}=65 \mathrm{~s} \pm 18.757, P c l o o^{g t / g t}=1.75 \pm 0.479, p^{* *}=0.0070, p^{\# \#}=0.0022 ;$ trial 16: $P c l o o^{w t / w t}=$

$109156.5 \mathrm{~s} \pm 16.775, P c l o{ }^{w t / g t}=68.75 \mathrm{~s} \pm 17.109$, Pclo $^{\text {gt/gt }}=2.25 \pm 0.25, p^{*}=0.0207, p^{\# \# \#}=0.009 ; \mathrm{n}=$

10924 animals per genotype; two-way ANOVA with Bonferroni correction).

1093 B) Grip strength task for $P c l o^{w t / w t}, P c l o^{w t / g t}$ and $P c l o^{g t / g t}$ rats for 9 trials over 2 days. No differences

1094 were found for forelimb grip strength between the groups. $\left(\right.$ Pclo $^{w t w t}=737.2 \pm 109.9 ;$ Pclo $^{w t / g t}=$

$1095621.2 \pm 93.46$, Pclo $^{g t / g t}=757.5 \pm 66.74 ; \mathrm{n}=4$ rats per genotype, one-way ANOVA, $\left.p=0.549\right)$.

1096 C) Ladder walk task for $P c l o^{w t / w t}, 859 \mathrm{Pclo}^{w t / g t}$ and $P c l o^{g t / g t}$ rats for 3 trials over 1 day. Pclo ${ }^{g t / g t}$ rats

1097 had a higher rate of stepping errors (ladder rung foot slips/misses) than $P c l o^{w t / w t}$ and $P c l o^{w t / g t}$ rats

1098 (forelimb error/step: Pclo ${ }^{w t / w t}=0.09 \pm 0.00925$, Pclo ${ }^{w t / g t}=0.148 \pm 0.0263$, Pclo $^{g t / g t}=0.297 \pm$

$10990.0145, \mathrm{n}=4$, one-way ANOVA, $p^{* *}=0.002, p^{\#}=0.0157$ (where $*=p$ value between Pclo ${ }^{w t / w}$

1100 and $P c l o^{g t / g t}$ and ${ }^{\#}=p$ value between $P c l o^{w t w t}$ and $\left.P c l o^{w t / g t}\right)$; hindlimb error/step: $P c l o^{w t / w t}=0.0563$

$1101 \pm 0.0221, P c l o o^{w t / g t}=0.0663 \pm 0.00877, P c l o o^{g t / g t}=0.238 \pm 0.0543, \mathrm{n}=4$; one-way ANOVA with

1102 Bonferroni correction, $\left.p^{*}=0.0135, p^{\#}=0.0186\right)$.

1103 D) Open field task for $P c l o^{w t / w t}, P c l o^{w t / g t}$ and $P c l o^{g t / g t}$ rats for 1 trial each over 1 day. Pclo ${ }^{g t / g t}$ rats

1104 performed fewer rearing behaviors than $P c l o^{w t / w t}$ and significantly less than $P c l o{ }^{w t / g t}$ rats in the

1105 perimeter sectors of the arena $\left(P c l o o^{w t / w t}=64.25 \pm 18.31\right.$ events; $P$ clo ${ }^{w t / g t}=76.25 \pm 11.3$ events;

$1106 P c l o^{g t / g t}=22 \pm 4.203$ events; $\mathrm{n}=4$ rats per genotype, one-way ANOVA with Bonferroni

1107 correction, $p^{\#}=0.0427$ (where ${ }^{\#}=p$ value between $P c l o o^{w t / w t}$ and $P c l o{ }^{w t / g t}$ ). Other behaviors such as

1108 crossing the open field and grooming were not different between the three groups (crossing:

$1109 P$ Pclo ${ }^{w t / w t}=178.3 \pm 29.68$ events; $P$ clo ${ }^{w t / g t}=164.5 \pm 45.51$ events; $P$ clo ${ }^{g t / g t}=235.5 \pm 27.11$ events,

$1110 \mathrm{n}=4$ rats per genotype, one-way ANOVA with Bonferroni correction, $p=0.828$; grooming:

$1111 P c l o o^{w t / w t}=2.75 \pm 0.75$ events; $P c l o^{w t g t}=2 \pm 1.08$ events; $P$ clo ${ }^{g t / g t}=5 \pm 1.155$ events, $\mathrm{n}=4$ rats

1112 per genotype, one-way ANOVA with Bonferroni correction, $p=0.488$ ) 
1113 E). Locomotor activity of $P c l o^{w t / w t}, P c l o{ }^{w t / g t}$ and $P c l o o^{g t / g t}$ rats during the $12 \mathrm{~h}$ dark phase. $P c l o{ }^{g t / g t}$

1114 rats covered a more than 50\% longer distance than $P c l o{ }^{w t / w t}$ and $P$ clo ${ }^{w t / g t}$ rats $\left(P c l o{ }^{w t / w t}=135.7 \pm\right.$

$111511.25, \mathrm{n}=10 ;$ Pclo $^{\text {wt/gt }}=129.3 \pm 10.44, \mathrm{n}=4 ;$ Pclo $^{\text {gt/gt }}=217.8 \pm 14.33, \mathrm{n}=12$; one-way

1116 ANOVA, $\left.p^{* * *}=0.0002\right)$. Data points are individual means over 15 nights.

1117 F) Performance of Pclo ${ }^{w t / w t}, P_{c l o}{ }^{w t / g t}$ and $P c l o{ }^{g t / g t}$ rats during the isometric pull-task (handle 1118 position $11 \mathrm{~mm}$ inside the cage). Only 4 out of $11 \mathrm{Pclo}^{\mathrm{gt} / \mathrm{gt}}$ rats succeeded at the $60 \mathrm{~g}$ force

1119 threshold and Pclo $^{\text {gt/gt }}$ rats pulled with a significantly lower success rate at all force thresholds as

1120 compared to Pclo ${ }^{w t / w t}$ and Pclo ${ }^{w t / g t} \cdot\left(30 \mathrm{~g}: P_{c}{ }^{w t / w t}=86.722 \pm 2.3, \mathrm{n}=10 ;\right.$ Pclo $^{w t / g t}=84.374 \pm$

$11216.324, \mathrm{n}=4 ; P c l o^{g t / g t}=61.044 \pm 5.928, \mathrm{n}=11 ; 2$ way ANOVA with Bonferroni correction, $p^{* *}=$

$11220.0018 ; 40 \mathrm{~g}:$ Pclo ${ }^{w t / w t}=83.603 \pm 2.86, \mathrm{n}=10 ;$ Pclo $^{w t / g t}=85.136 \pm 7.373, \mathrm{n}=4 ;$ Pclo ${ }^{g t / g t}=47.804 \pm$

$11236.897, \mathrm{n}=10$; two-way ANOVA with Bonferroni correction, $p^{* * * *}<0.0001, p^{\# \# \#}=0.0007 ; 50 \mathrm{~g}$ :

$1124 P$ Pclo ${ }^{w t / w t}=80.49 \pm 3.442, \mathrm{n}=10 ; P c l o^{w t / g t}=71.930 \pm 8.695, \mathrm{n}=4 ; P c l o^{g t / g t}=33.647 \pm 6.802, \mathrm{n}=$

1125 10; two-way ANOVA with Bonferroni correction, $p^{* * * *}<0.0001, p^{\# \# \#}=0.0005$ 60g: Pclo ${ }^{\text {wt/wt }}=$

$112669.186 \pm 4.99, \mathrm{n}=10 ; P_{c}{ }^{w t g t}=70.409 \pm 10.181, \mathrm{n}=3 ; P c l o{ }^{g t / g t}=30.003 \pm 11.359, \mathrm{n}=4 ; 2$ way

1127 ANOVA with Bonferroni correction, $p^{* * *}=0.0004, p^{\# \#}=0.0056$ ).

1128 Error bars represent SEM. Data points represent individual rats. 


\section{Figure 1}

A

B

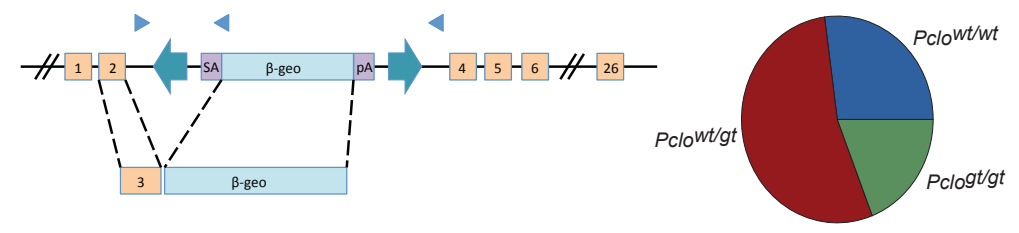

C

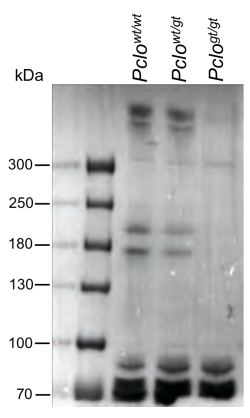

G

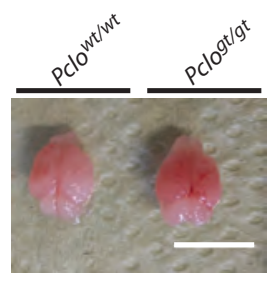

$J$

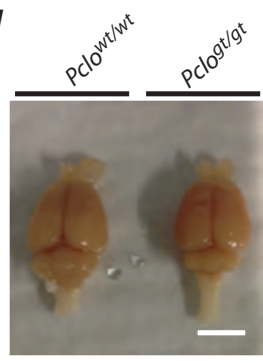

$D$
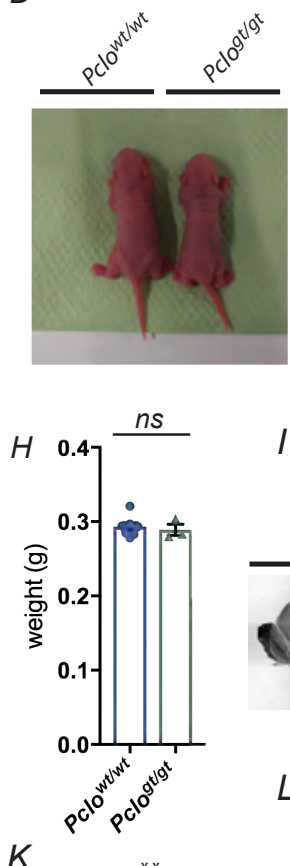

K

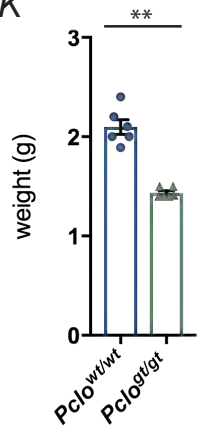

E

F
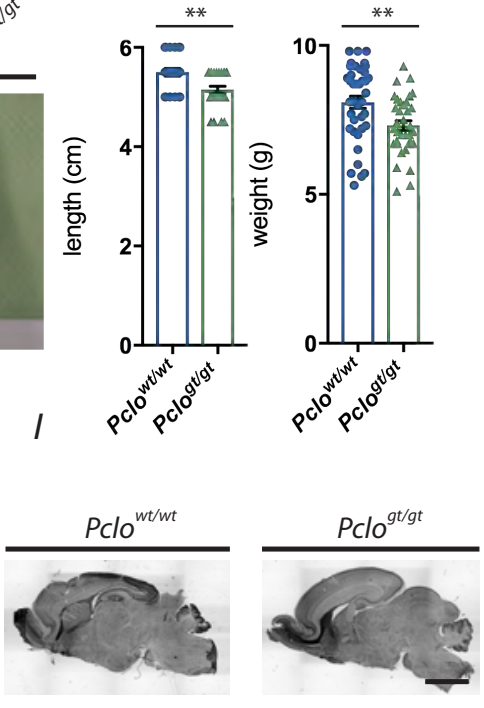

$L \quad$ Pclo ${ }^{w t / w t}$

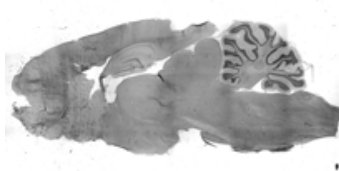

Pclo ${ }^{g t / g t}$

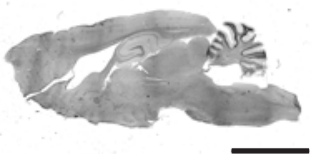




\section{Figure 2}

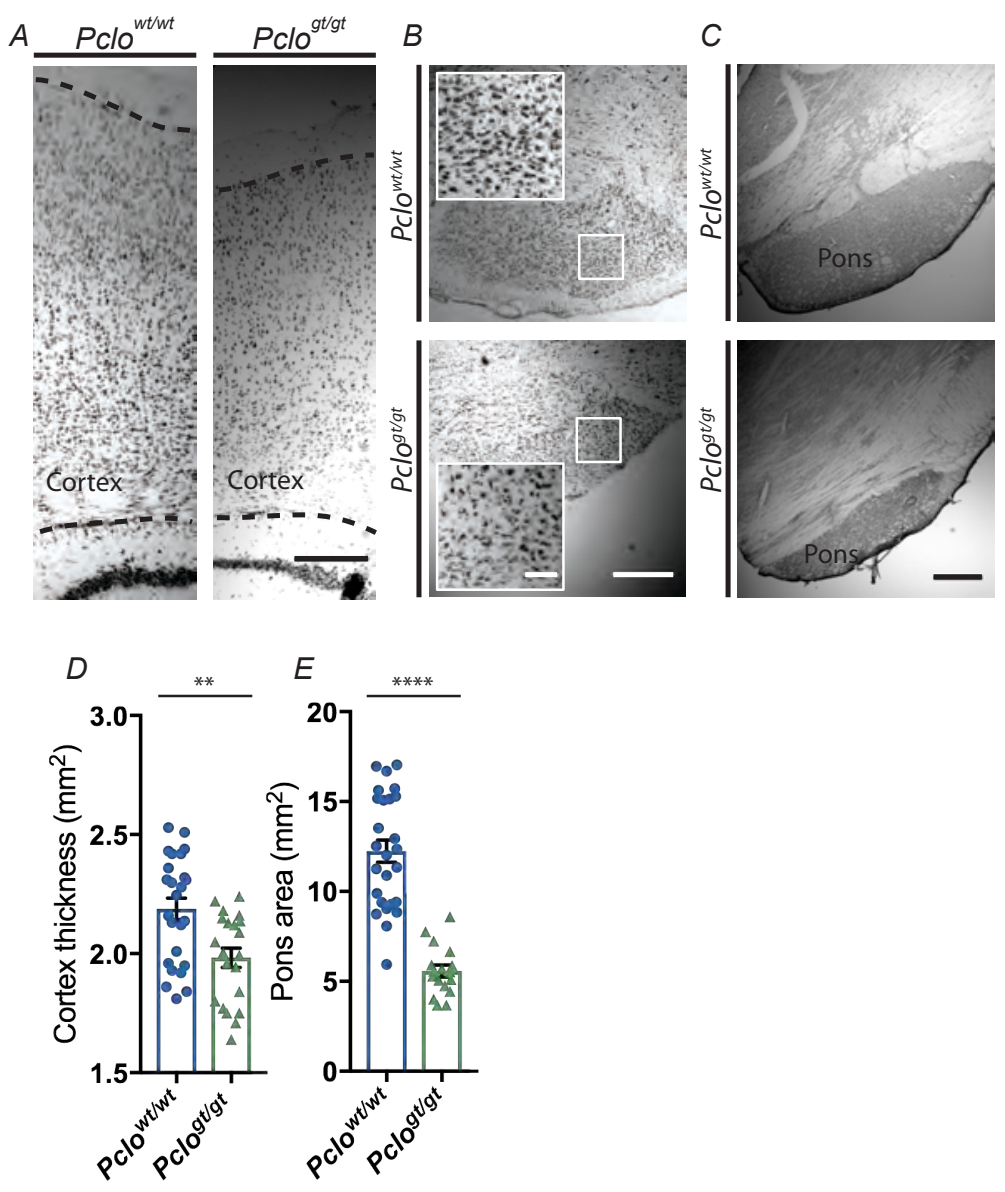




\section{Figure 3}

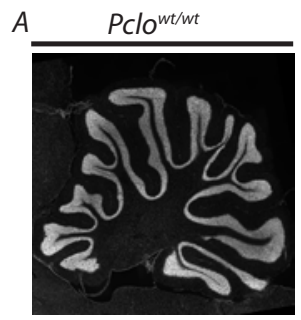

B

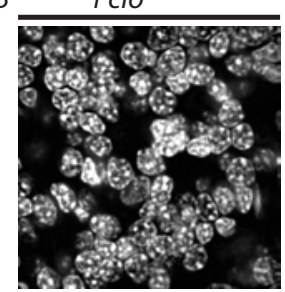

C

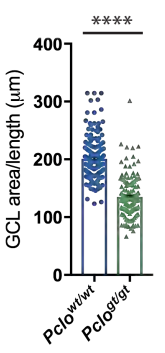

Pclo ${ }^{g t / g t}$

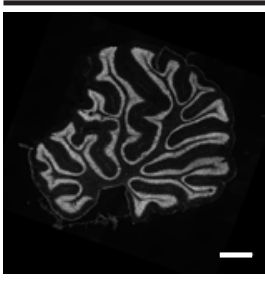

$\mathrm{PClO}^{\mathrm{gt} / \mathrm{gt}}$

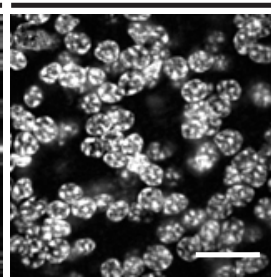

$D \quad * *$
$G \quad * * * * \quad H$

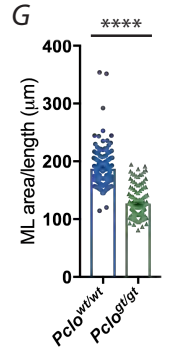

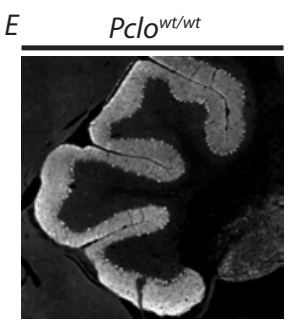

F $\quad \mathrm{PClO}^{w t / w t}$
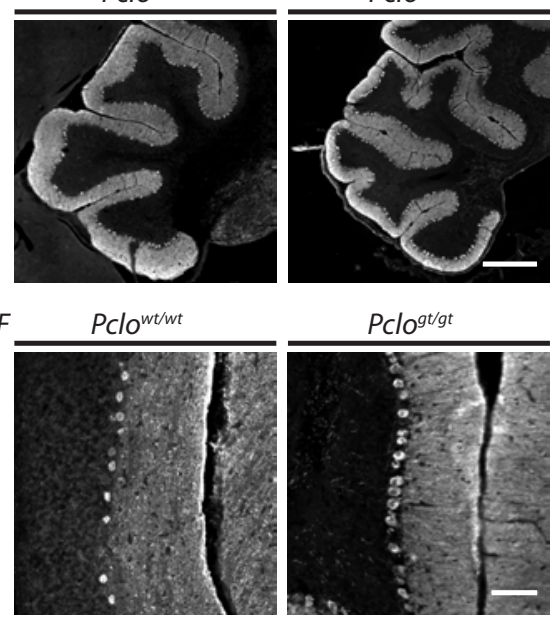

$\mathrm{PClO}^{\mathrm{gt} / \mathrm{gt}}$
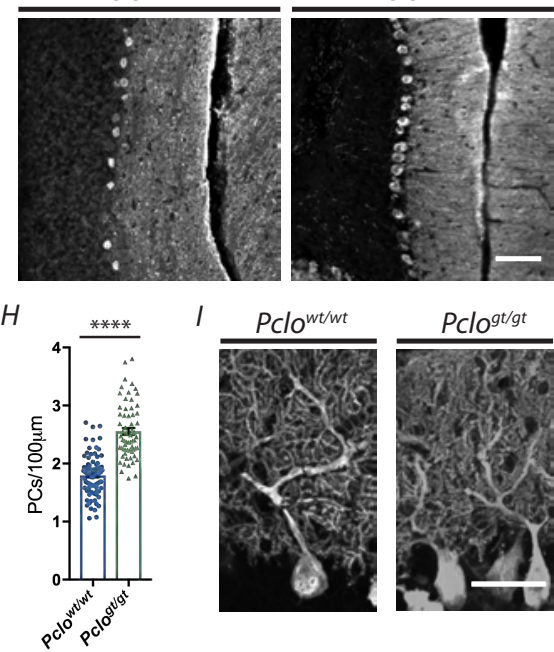

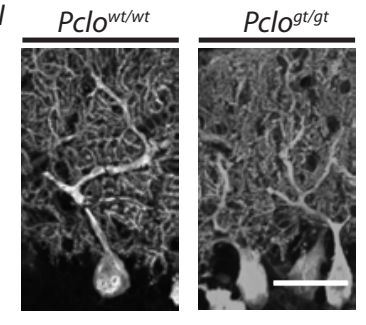


Figure 4
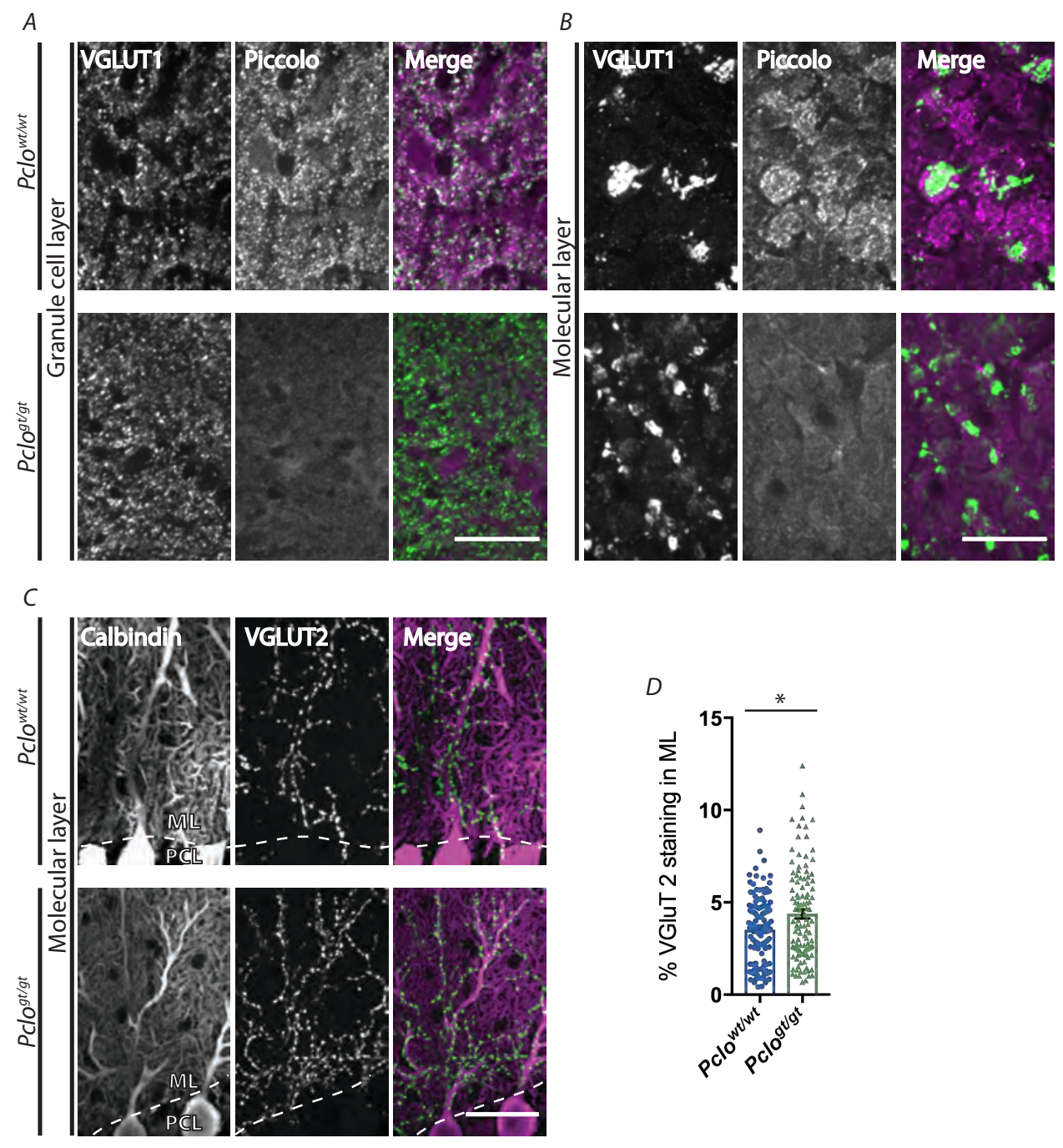
Figure 5

$A$
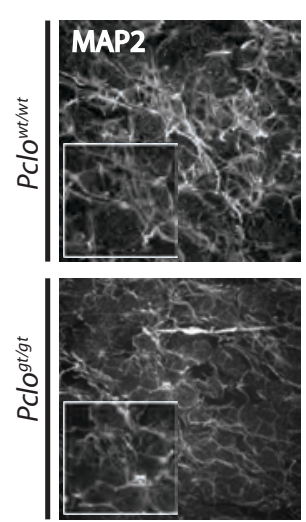

$B$
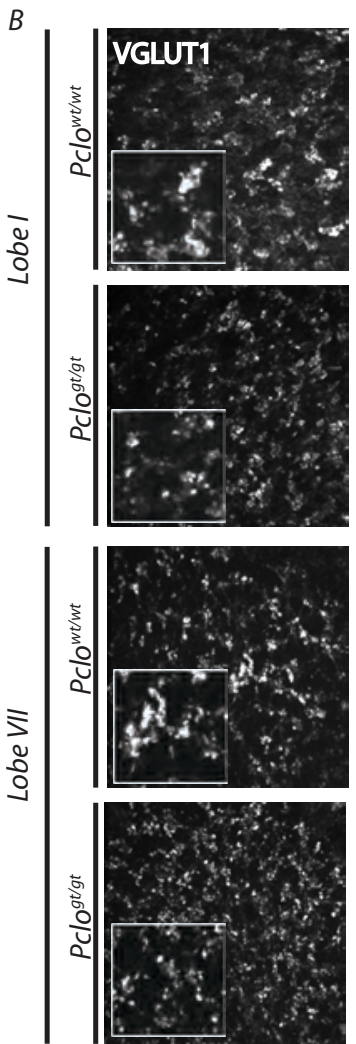
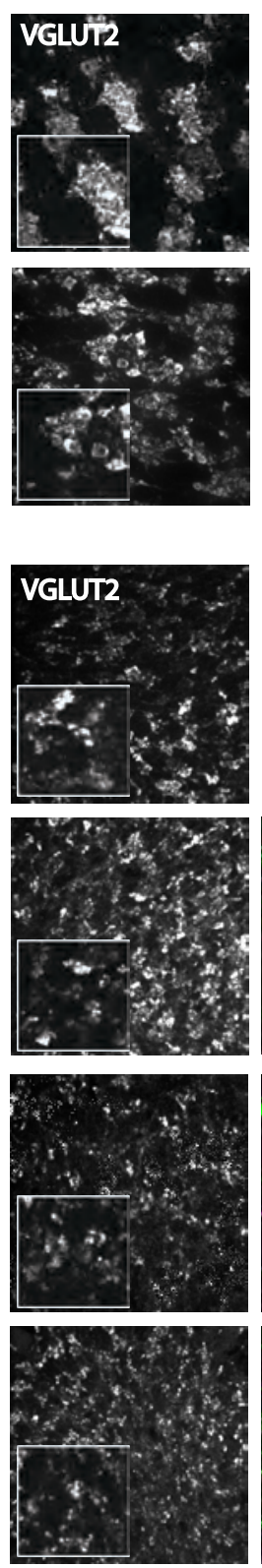

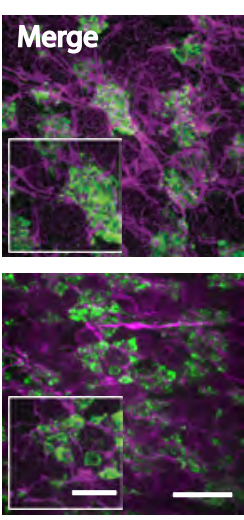

C
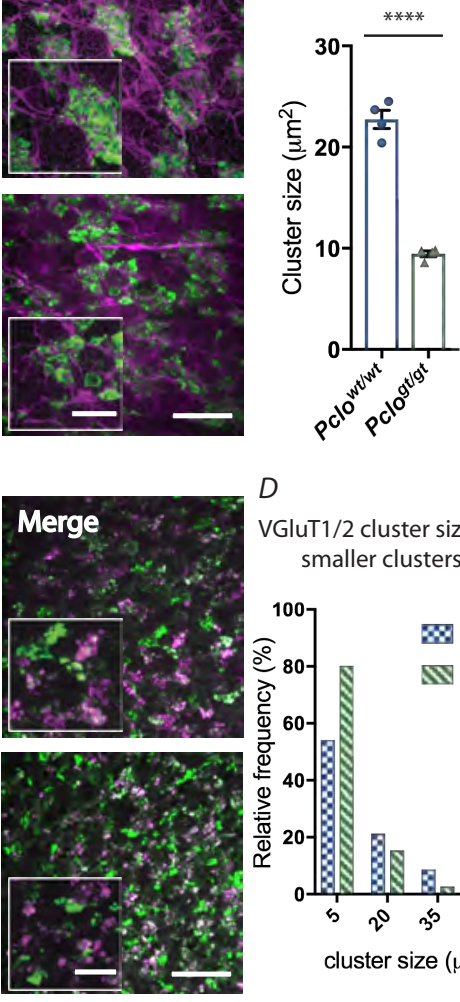

VGluT1/2 cluster size distribution smaller clusters: 5-50 $\mathrm{m}^{2}$
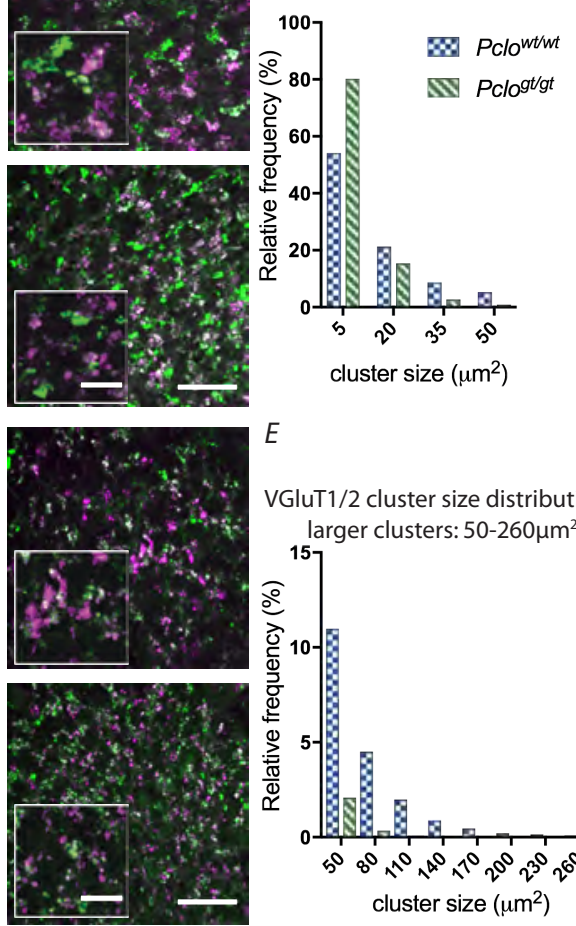

VGluT1/2 cluster size distribution

larger clusters: 50-260 $\mathrm{m}^{2}$

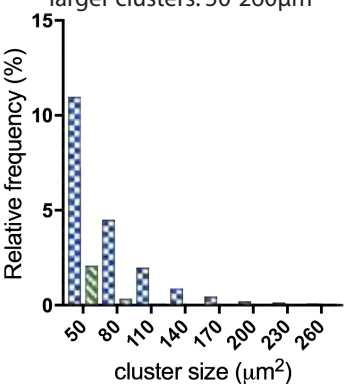


Figure 6
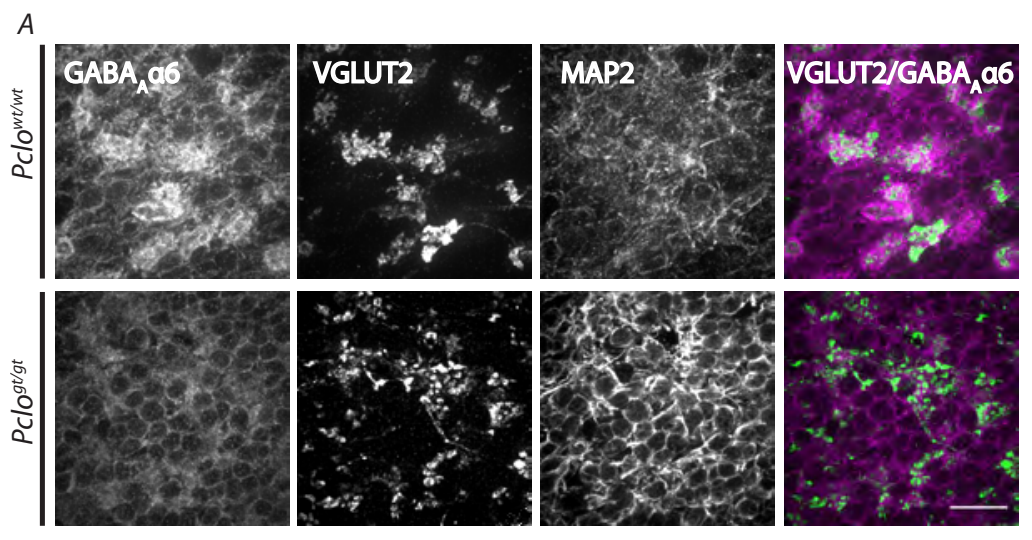

$B$

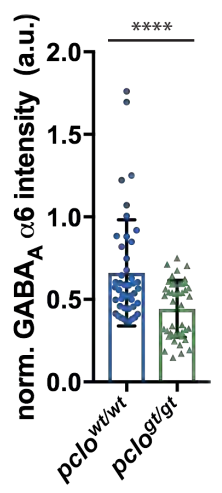




\section{Figure 7}
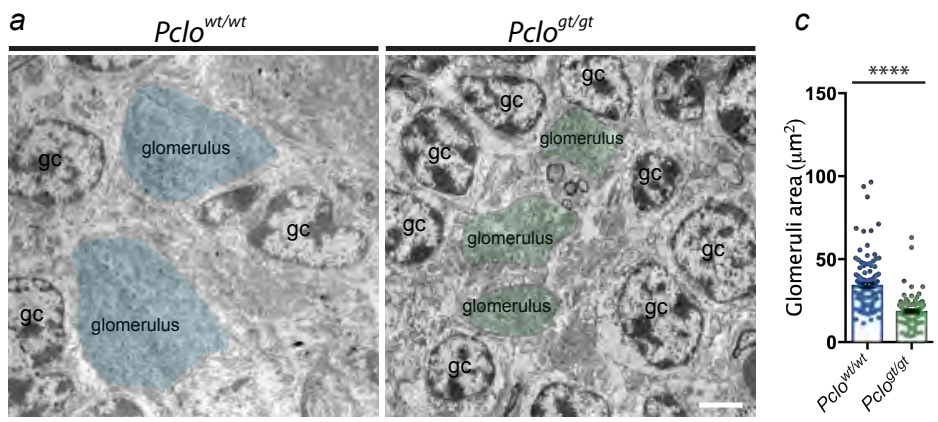

$d$

$b$

Pclo ${ }^{g t / g t}$
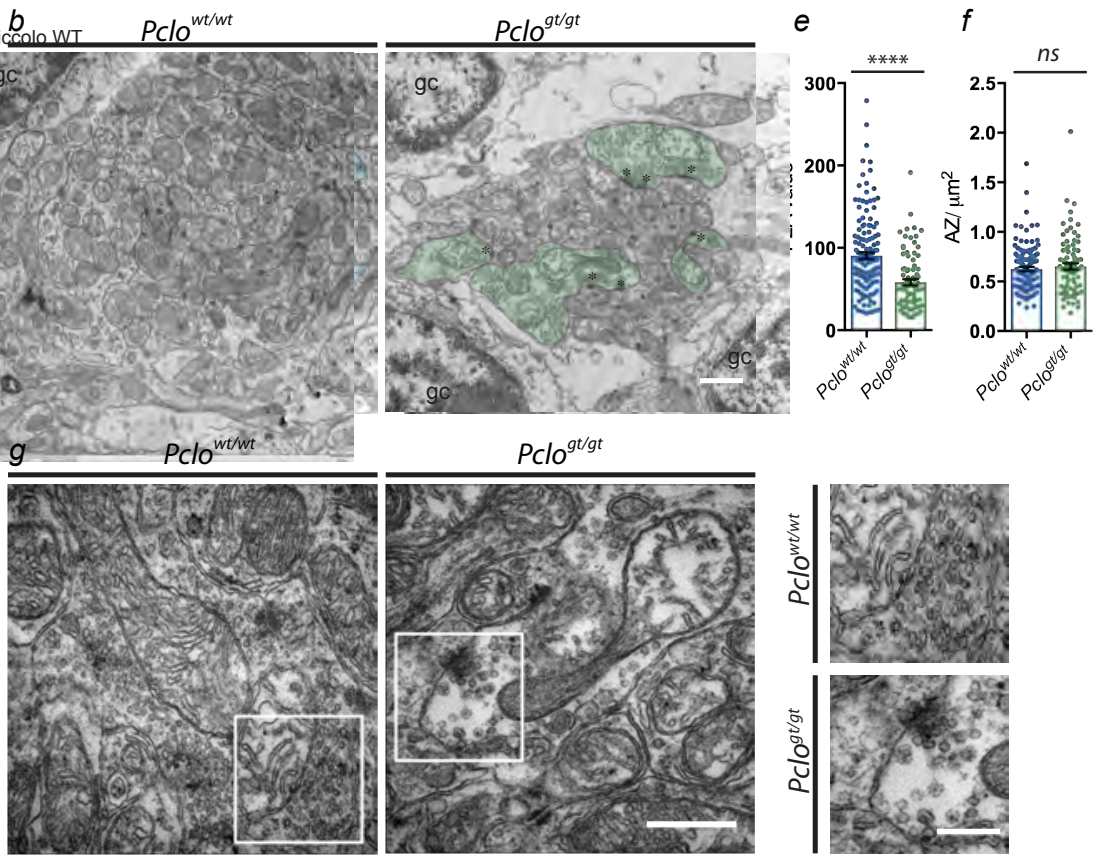


\section{Figure 8}
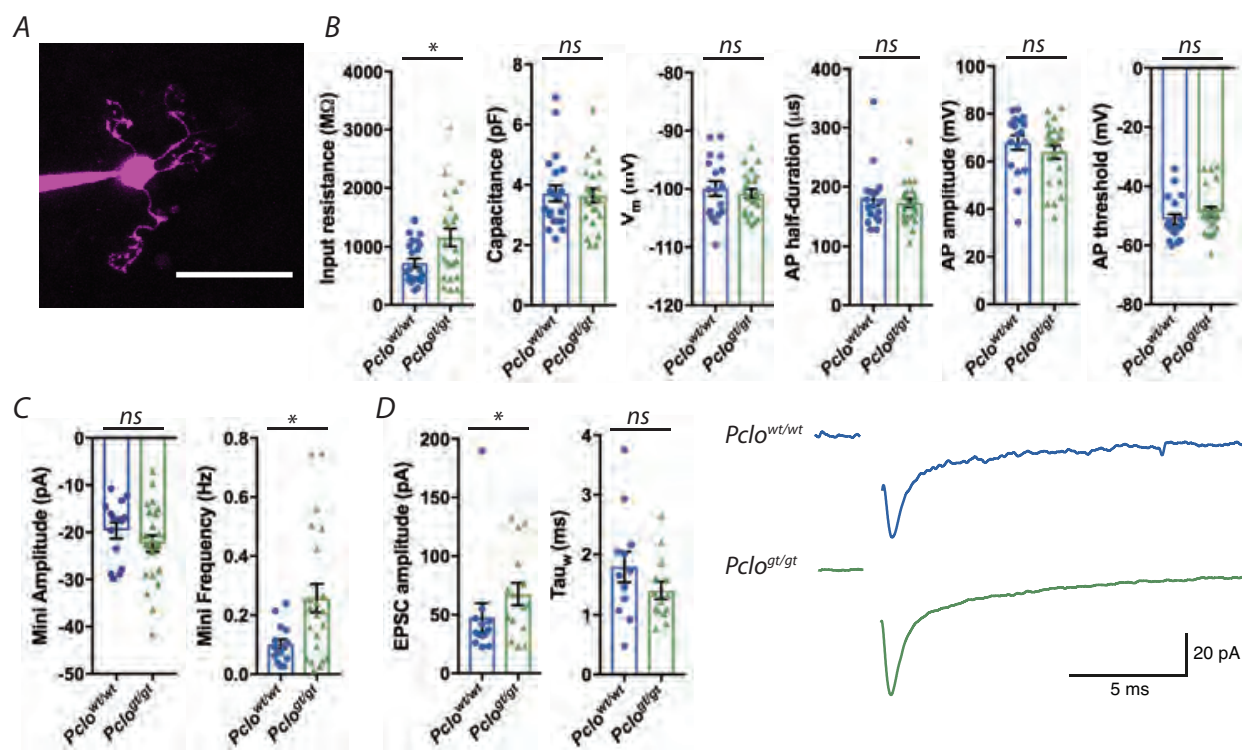

$\mathrm{PClO}^{\mathrm{wt} / \mathrm{wt}} \mathrm{nm}$

Pclogtgt

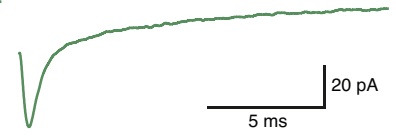


bioRxiv preprint dol: https://doi.org/10.1101/774422; this version posted September 25, 2019. The copyright holder for this preprint (which was

not certified by peer review) is the author/funder. All rights reserved. No reuse allowed without permission.

Figure 9

$A$

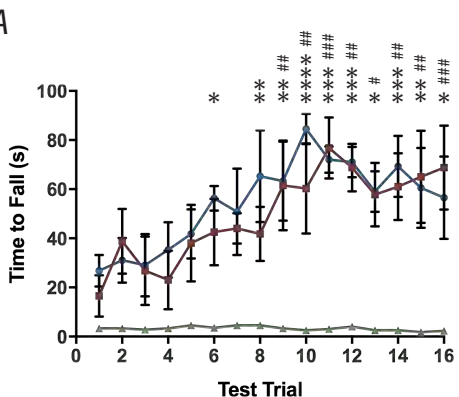

$D$

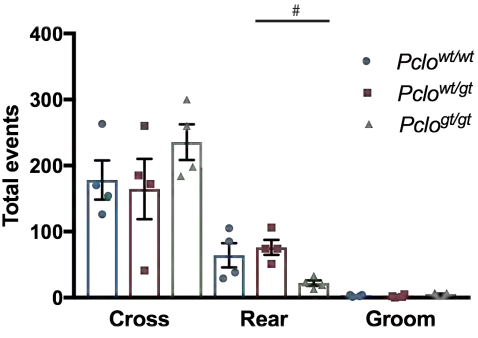

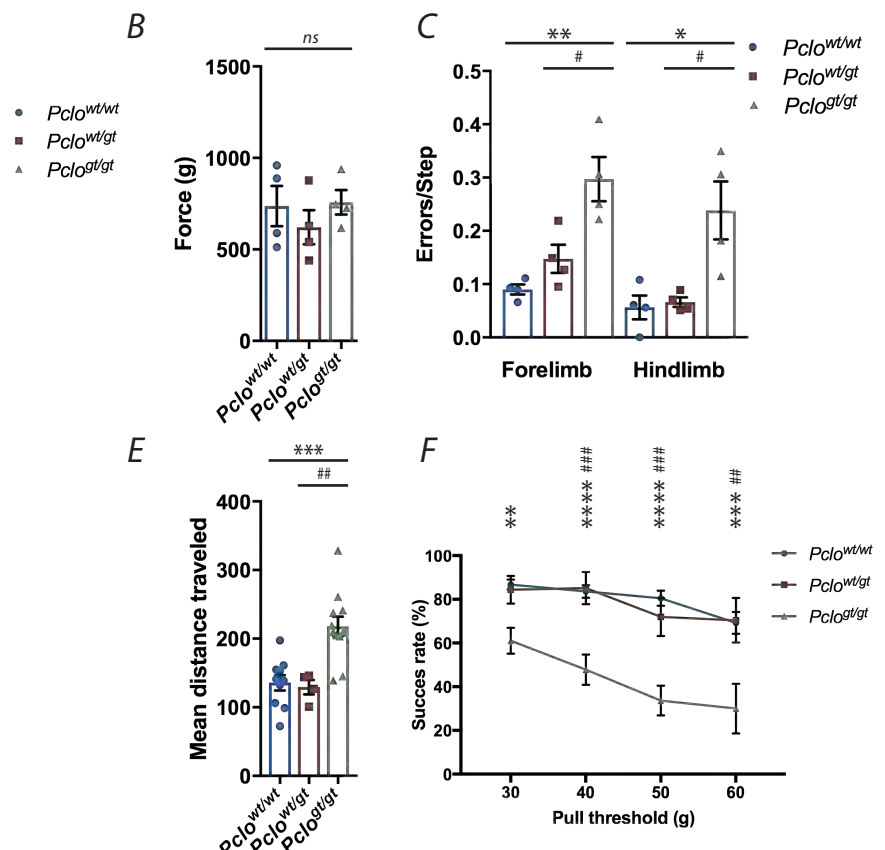

